\title{
How H-bonding Affects Aromaticity of the Ring in Variously Substituted Phenol Complexes with Bases.
}

\author{
Tadeusz M. Krygowski , Halina Szatytowicz,"* and Joanna E. Zachara ${ }^{\dagger}$ \\ 'Department of Chemistry, Warsaw University, Pasteura 1, 02-093 Warsaw, Poland, \\ Department of Chemistry, Warsaw University of Technology, Noakowskiego 3, 00-664 Warsaw, \\ Poland \\ halina@ chemix.ch.pw.edu.pl
}

\section{Supporting Information}

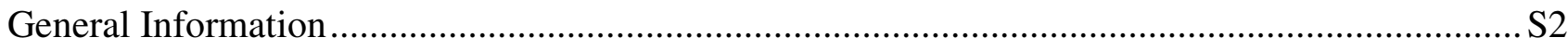

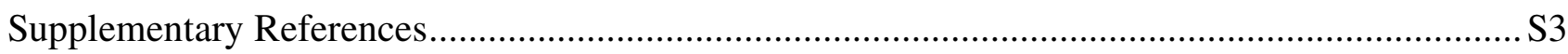




\section{General Information}

Geometries of 664 rings of H-bonded complexes of variously substituted phenol derivatives with various oxygen and nitrogen bases were retrieved from the 5.25 version (November 2003, updated January 2004) of The Cambridge Crystallographic Database (CSD) using CCDC software with the following restrictions: (1) the searches were performed for structures containing substituted phenol and a nitrogen or oxygen base having an intermolecular contact between oxygen of the hydroxyl group of phenol and the nearest $\mathrm{O}-$ or $\mathrm{N}-$ atom in base equal to or less than the sum of their van der Waals radii $^{1}$; (2) the searches were restricted to structure measurements with the reported mean estimated standard deviation (e.s.d.) of the CC bond $-0.005 \AA$ (corresponds to AS flag =1). The data were retrieved for polysubstituted phenols (by any of the following substituents: halogen, $-\mathrm{NO}_{2},-\mathrm{NH}_{2}$, $-\mathrm{COOH},-\mathrm{CONH}_{2},-\mathrm{COCH}_{3},-\mathrm{COOCH}_{3},-\mathrm{CHO},-\mathrm{OH},-\mathrm{SH},-\mathrm{N}=\mathrm{O},-\mathrm{Me},-i \mathrm{Pr},-t \mathrm{Bu},-\mathrm{Ph},-\mathrm{CPh}_{3}$, $\left.-\mathrm{SiMe}_{3},-\mathrm{SO}_{3} \mathrm{H},-\mathrm{H}\right)$ interacting with $\mathrm{N}-$ or $\mathrm{O}-$ atom in base. Sometimes the molecules of the solvent were present in the structure lattice.

(1) Bondi, A. Van der Waals Volumes and Radii. J. Phys. Chem. 1964, 68, 441-451. 
Supporting Information for The Journal of Chemical Information and Computer Science

\section{Supplementary references}

ABEKUN resorcinol trans-1,2-bis(4-pyridyl)ethylene; C12 H10 N2,C6 H6 O2;

L.R.MacGillivray, J.L.Reid, J.A.Ripmeester; J.Am.Chem.Soc., 122, 7817, 2000.

ABELIC resorcinol trans-1,2-bis(2-pyridyl)ethylene; C6 H6 O2,C12 H10 N2;

L.R.MacGillivray, J.L.Reid, J.A.Ripmeester; J.Am.Chem.Soc., 122, 7817, 2000.

ACIFAT 4-Aminobenzenesulfonamide 3,5-dinitrosalicylate; C6 H9 N2 O2 S1 1+,C7 H3 N2

O7 1-; G.Smith, U.D.Wermuth, J.M.White; Acta Crystallogr.,Sect.E:

Struct.Rep.Online, 57, o1036, 2001.

ACOYOG trans-1,2-bis(4-pyridyl)ethane resorcinol; C12 H12 N2,C6 H6 O2;

G.S.Papaefstathiou, L.R.MacGillivray; Organic Letters, 3, 3835, 2001.

ACOYUM trans-1,2-bis(4-pyridyl)ethane 4-chlororesorcinol; C12 H12 N2,C6 H5 Cl1 O2;

G.S.Papaefstathiou, L.R.MacGillivray; Organic Letters, 3, 3835, 2001.

ACOZAT trans-1,2-bis(4-pyridyl)ethane 4,6-dichlororesorcinol; C12 H12 N2,C6 H4 Cl2 O2;

G.S.Papaefstathiou, L.R.MacGillivray; Organic Letters, 3, 3835, 2001.

AHUYIL 5,6-dihydro-2-methyl-4H-thieno[2,3-f][1,2,4]triazolo[1,5-a]azepine picrate; C10

H12 N3 S1 1+,C6 H2 N3 O7 1-; Quanrui Wang, Zheng Li, Haiyan Yang, Feng Li,

Zongbiao Ding, Fenggang Tao; Synthesis, , 1231, 2003.

AJAGOH Piperazine-2,5-dione 2,5-dichloro-1,4-hydroquinone; C4 H6 N2 O2,C6 H4 Cl2 O2;

Tzy-Jiun M.Luo, G.T.R.Palmore; Cryst.Growth Des., 2, 337, 2002.

AJAGUN Piperazine-2,5-dione 2,5-dihydroxybenzoic acid; C4 H6 N2 O2,C7 H6 O4; Tzy-

Jiun M.Luo, G.T.R.Palmore; Cryst.Growth Des., 2, 337, 2002.

AJAHEY Piperazine-2,5-dione bis(5-chloro-2-hydroxybenzoic acid); C4 H6 N2 O2,2(C7 H5

Cl1 O3); Tzy-Jiun M.Luo, G.T.R.Palmore; Cryst.Growth Des., 2, 337, 2002.

AJEBIA Pyridinium 3,5-dinitrosalicylate 3,5-dinitrosalicylic acid; C5 H6 N1 1+,C7 H3 N2

O7 1-,C7 H4 N2 O7; G.Smith, U.D.Wermuth, P.C.Healy, J.M.White; Aust.J.Chem., 56, 707, 2003. 
AJEBOG 4-Cyanopyridinium 3,5-dinitrosalicylate; C6 H5 N2 1+,C7 H3 N2 O7 1-; G.Smith, U.D.Wermuth, P.C.Healy, J.M.White; Aust.J.Chem., 56, 707, 2003.

AJULEW 4-Dimethylaminopyridinium picrate; C7 H11 N2 1+,C6 H2 N3 O7 1-; N.Vembu, M.Nallu, J.Garrison, W.J.Youngs; Acta Crystallogr.,Sect.E: Struct.Rep.Online, 59, o913, 2003.

AMBSAM10 5-Ethyl-5-isoamylbarbituric acid-salicylamide complex; C11 H18 N2 O3,C7 H7 N1

O2; I.-N.Hsu, B.M.Craven; Acta Crystallogr.,Sect.B:

Struct.Crystallogr.Cryst.Chem., 30, 843, 1974.

AMNPHA 2-Amino-5-nitrophenol; C6 H6 N2 O3; M.Haisa, S.Kashino, T.Kawashima; Acta Crystallogr.,Sect.B: Struct.Crystallogr.Cryst.Chem., 36, 1598, 1980.

AMPCPL Ammonium picrate 1,10-phenanthroline; C12 H8 N2,C6 H2 N3 O7 1-,H4 N1 1+; C.L.Jones, G.H.Milburn, L.Sawyer, D.L.Hughes; Acta Crystallogr.,Sect.B: Struct.Crystallogr.Cryst.Chem., 37, 1548, 1981.

AMPHOM02 2-Aminophenol; C6 H7 N1 O1; J.D.Korp, I.Bernal, L.Aven, J.L.Mills; J.Cryst.Mol.Struct., 11, 117, 1981.

AMPHOM03 2-Aminophenol; C6 H7 N1 O1; F.H.Allen, V.J.Hoy, J.A.K.Howard, V.R.Thalladi, G.R.Desiraju, C.C.Wilson, G.J.McIntyre; J.Am.Chem.Soc., 119, 3477, 1997.

AMPICR01 Ammonium picrate; C6 H2 N3 O7 1-,H4 N1 1+; J.M.Harrowfield, B.W.Skelton, A.H.White; Aust.J.Chem., 48, 1311, 1995.

BAHPEG bis(Aminoguanidinium) styphnate hemihydrate; 2(C1 H7 N4 1+),C6 H1 N3 O8 2,0.5(H2 O1); Li Yang, Tong-Lai Zhang, Jian-Guo Zhang, Yong-Ming Yang, ChangGen Feng, Kai-Bei Yu; Youji Huaxue(Chin.)(Chin.J.Org.Chem.), 21, 1132, 2001. BAHPIK bis(N-Amino-N'-ammoniourea) styphnate; 2(C1 H7 N4 O1 1+),C6 H1 N3 O8 2-; Li Yang, Tong-Lai Zhang, Jian-Guo Zhang, Yong-Ming Yang, Chang-Gen Feng, KaiBei Yu; Youji Huaxue(Chin.)(Chin.J.Org.Chem.), 21, 1132, 2001. 
Supporting Information for The Journal of Chemical Information and Computer Science

BEBPIH 4b,5,15b,16-Tetrahydrodibenzo(3,4:7,8)(1,5)diazocino(2,1-b;C28 H22 N4 2+,2(C6

H2 N3 O7 1-); P.G.Owston, L.S.Shaw, P.A.Tasker; Chem.Commun., , 17, 1982.

BEBPIH10 4b,5,15b,16-Tetrahydrodibenzo(3,4:7,8)(1,5)diazocino(2,1-b;C28 H22 N4 2+,2(C6

H2 N3 O7 1-); P.G.Owston, L.S.Shaw; Acta Crystallogr.,Sect.B: Struct.Sci., 44, 39, 1988.

BEQVUO 5-Hydroxyisophthalic acid 4,4'-trimethylenedipyridine; C13 H15 N2 1+,C8 H5 O5

1-; P.S.Wheatley, A.J.Lough, G.Ferguson, C.Glidewell; Acta Crystallogr.,Sect.C:

Cryst.Struct.Commun., 55, 1486, 1999.

BEQWAV bis(3,5-Dihydroxybenzoic acid) tris(4,4'-bipyridine); 3(C10 H8 N2),2(C7 H6 O4);

P.S.Wheatley, A.J.Lough, G.Ferguson, C.Glidewell; Acta Crystallogr.,Sect.C:

Cryst.Struct.Commun., 55, 1489, 1999.

BERBAB 4-(Triphenylmethyl)phenol triphenylphosphine oxide; C25 H20 O1,C18 H15 O1 P1;

R.K.R.Jetti, H.-C.Weiss, V.R.Thalladi, R.Boese, A.Nangia, G.R.Desiraju; Acta Crystallogr.,Sect.C: Cryst.Struct.Commun., 55, 1530, 1999.

BESKAL 2,5-Dihydroxybenzoic acid; C7 H6 O4; M.Haisa, S.Kashino, S.-I.Hanada,

K.Tanaka, S.Okazaki, M.Shibagaki; Acta Crystallogr.,Sect.B:

Struct.Crystallogr.Cryst.Chem., 38, 1480, 1982.

BIDLOP m-Hydroxybenzoic acid; C7 H6 O3; G.V.Gridunova, N.G.Furmanova,

Yu.T.Struchkov, Z.I.Ezhkova, L.P.Grigor'eva, B.A.Chayanov;

Kristallografiya(Russ.)(Crystallogr.Rep.), 27, 267, 1982.

BIJDON03 3,4-Dihydroxybenzoic acid monohydrate; C7 H6 O4,H2 O1; V.Horneffer,

K.Dreisewerd, H.-C.Ludemann, F.Hillenkamp, M.Lage, K.Strupat; Int.J.Mass

Spectrom.Ion.Process., 185, 859, 1999.

BIKWEX diammonium styphnate; C6 H1 N3 O8 2-,2(H4 N1 1+); Lu Chunhua, Zhang

Tonglai, Ren Lingbai, Zhang Zhigang, Yu Kaibei; Beijing Ligong

Dax.Xuebao(Chin.)(J.Beijing Inst.Tech.), 19, 25, 1999. 
BOCNEM 2,4,6-Trinitro-1,3-benzenediol hydrate; C6 H3 N3 O8,0.67(H2 O1); M.A.PierceButler; Acta Crystallogr.,Sect.B: Struct.Crystallogr.Cryst.Chem., 38, 3097, 1982.

BOCNIQ 2,4,6-Trinitro-1,3,5-benzenetriol hydrate; C6 H3 N3 O9,0.67(H2 O1); M.A.Pierce-

Butler; Acta Crystallogr.,Sect.B: Struct.Crystallogr.Cryst.Chem., 38, 3097, 1982.

BODSAO Resorcinol urea; C6 H6 O2,C1 H4 N2 O1; M.Pickering, R.W.H.Small; Acta Crystallogr.,Sect.B: Struct.Crystallogr.Cryst.Chem., 38, 3161, 1982.

BOJDAF Calebassinine-1 picrate; C19 H23 N2 O2 1+,C6 H2 N3 O7 1-; A.Guggisberg, R.Prewo, J.H.Bieri, M.Hesse; Helv.Chim.Acta, 65, 2587, 1982.

BOQXEK (8-Quinolinyl)urea 3,5-dinitrosalicylate; C10 H10 N3 O1 1+,C7 H3 N2 O7 1-; G.Smith, R.C.Bott, U.D.Wermuth; Acta Crystallogr.,Sect.E: Struct.Rep.Online, 57, o895, 2001.

BUNGIA Methyl 2,4-dihydroxy-3,6-dimethylbenzoate; C10 H12 O4; L.Brehm, H.StoeckliEvans, R.Tabacchi, H.-B.Burgi; Helv.Chim.Acta, 66, 824, 1983.

CABQIF tris(Hexadecyl-trimethylammonium) tribromide p-hydroxybenzoic acid monohydrate; 3(C19 H42 N1 1+),3(Br1 1-),2(C7 H6 O3),H2 O1; K.Sawada, T.Kitamura, Y.Ohashi, N.Iimura, H.Hirata; Bull.Chem.Soc.Jpn., 71, 2109, 1998.

CABWAD Hexadecyl-trimethylammonium bromide hydroquinone dihydrate; C19 H42 N1 1+,Br1 1-,C6 H6 O2,2(H2 O1); K.Sawada, T.Kitamura, Y.Ohashi, N.Iimura, H.Hirata; Bull.Chem.Soc.Jpn., 71, 2109, 1998.

CACDAM 2,3-Dihydroxybenzoic acid; C7 H6 O4; N.Okabe, H.Kyoyama; Acta Crystallogr.,Sect.E: Struct.Rep.Online, 57, o1224, 2001.

CATCOL12 Catechol; C6 H6 O2; H.Wunderlich, D.Mootz; Acta Crystallogr.,Sect.B: Struct.Crystallogr.Cryst.Chem., 27, 1684, 1971.

CATCOL13 1,2-Benzenediol; C6 H6 O2; F.R.Fronczek, Kyu Kwang Kim, R.M.Strongin; Private Communication, , , 2002. 
CAXNOE 4-Nitrophenol 4-methylpyridine; C6 H5 N1 O3,C6 H7 N1; Zhi Min Jin, Yuan Jiang Pan, Duan Jun Xu, Yuan Zhi Xu; Acta Crystallogr.,Sect.C: Cryst.Struct.Commun., 56, e69, 2000.

CEKBOJ 2,9-Dimethyl-1,10-phenanthroline resorcinol; C14 H12 N2,C6 H6 O2;

W.H.Watson, J.Galloy, F.Vogtle, W.M.Muller; Acta Crystallogr.,Sect.C:

Cryst.Struct.Commun., 40, 200, 1984.

CEKXUL 1,4,7,10,13,16-Hexaoxacyclo-octadecane bis(2,4-dinitrophenol) clathrate dihydrate; C12 H24 O6,2(C6 H4 N2 O5),2(H2 O1); M.R.Caira, W.H.Watson, F.Vogtle,

W.Muller; Acta Crystallogr.,Sect.C: Cryst.Struct.Commun., 40, 491, 1984.

CENKOV N,2alpha-Dimethyl-8alpha-t-butyl-cis-decahydroquinoline picrate; C15 H30 N1

1+,C6 H2 N3 O7 1-; K.D.Onan, F.W.Vierhapper; Tetrahedron Lett., 25, 799, 1984.

CIJTEU N-Benzyl-cis-4,5-tetramethylenetetrahydro-1,3-oxazinium picrate; C15 H22 N1 O1 1+,C6 H2 N3 O7 1-; F.Fulop, G.Bernath, G.Argay, A.Kalman, P.Sohar;

Tetrahedron, 40, 2053, 1984.

CIJXOI10 3,8-Dimethyl-2-azoniabicyclo(2.2.2)oct-2-en-5-one picrate; C9 H14 N1 O1 1+,C6

H2 N3 O7 1-; G.B.Robertson, U.Tooptakong; Acta Crystallogr.,Sect.C:

Cryst.Struct.Commun., 41, 1332, 1985.

CIKRAP bis(2,5-Dimethyl-1,4-benzoquinone) 2,5-dimethyl-1,4-dihydroxybenzene; 2(C8 H8

O2),C8 H10 O2; A.O.Patil, S.R.Wilson, D.Y.Curtin, I.C.Paul; J.Chem.Soc.,Perkin

Trans.2, , 1107, 1984.

CILJIQ 2-Bromo-5-hydroxybenzaldehyde; C7 H5 Br1 O2; A.Matos Beja, J.A.Paixao, M.Ramos Silva, L.Alte da Veiga, A.M.d'A.Rocha Gonsalves, A.C.Serra; Acta Crystallogr.,Sect.C: Cryst.Struct.Commun., 56, 354, 2000.

CIPVUS N,N,N',N'-Tetramethylpiperazine-di-ium bis(2,4-dinitrophenolate) dihydrate; C8 H20 N2 2+,2(C6 H3 N2 O5 1-),2(H2 O1); F.Iwasaki, K.Mutai; Bull.Chem.Soc.Jpn., 57, 1295, 1984. 
CIRTEC01 1,4,7,10,13,16-Hexaoxacyclo-octadecane bis(dichloropicric acid) dihydrate; C12 H24 O6,2(C6 H1 Cl2 N3 O7),2(H2 O1); D.Britton, M.K.Chantooni Junior, I.M.Kolthoff; Acta Crystallogr.,Sect.C: Cryst.Struct.Commun., 44, 303, 1988.

CIRTEC02 1,4,7,10,13,16-Hexaoxacyclo-octadecane bis(dichloropicric acid) dihydrate; C12 H24 O6,2(C6 H1 Cl2 N3 O7),2(H2 O1); D.Britton, M.K.Chantooni Junior, I.M.Kolthoff; Acta Crystallogr.,Sect.C: Cryst.Struct.Commun., 44, 303, 1988.

CISCOW 2,5-Dimethylbenzoquinone bis(hydroquinone); C8 H8 O2,2(C6 H6 O2); A.O.Patil, D.Y.Curtin, I.C.Paul; J.Am.Chem.Soc., 106, 4010, 1984.

COBTUI 2,6-Pyrido-27-crown-9 picric acid; C21 H36 N1 O8 1+,C6 H2 N3 O7 1-; C.J.van Staveren, H.J.den Hertog Junior, D.N.Reinhoudt, J.W.H.M.Uiterwijk, L.Kruise, S.Harkema; Chem.Commun., , 1409, 1984.

COBTUI10 (2,6-Pyridinium)-1,4,7,10,13,16,19,22,25-azoctaoxacycloheptacosane picrate; C21 H36 N1 O8 1+,C6 H2 N3 O7 1-; J.W.H.M.Uiterwijk, C.J.van Staveren, D.N.Reinhoudt, H.J.den Hertog Junior, L.Kruise, S.Harkema; J.Org.Chem., 51, $1575,1986$.

CUTVOC meso-5,5,7,12,12,14-Hexamethyl-1,4,8,11-tetra-azacyclotetradecane 3hydroxybenzoate methanol solvate; C16 H38 N4 2+,2(C7 H5 O3 1-),2(C1 H4 O1); R.M.Gregson, C.Glidewell, G.Ferguson, A.J.Lough; Acta Crystallogr.,Sect.B: Struct.Sci., 56, 39, 2000.

CUTXIY meso-5,5,7,12,12,14-Hexamethyl-1,4,8,11-tetra-azacyclotetradecane bis(4hydroxybenzoate) methanol solvate; C16 H38 N4 2+,2(C7 H5 O3 1-),C1 H4 O1; R.M.Gregson, C.Glidewell, G.Ferguson, A.J.Lough; Acta Crystallogr.,Sect.B: Struct.Sci., 56, 39, 2000.

CUTXUK meso-5,5,7,12,12,14-Hexamethyl-1,4,8,11-tetra-azacyclotetradecane bis(3,5dihydroxybenzoate); C16 H38 N4 2+,2(C7 H5 O4 1-); R.M.Gregson, C.Glidewell, G.Ferguson, A.J.Lough; Acta Crystallogr.,Sect.B: Struct.Sci., 56, 39, 2000. 
CUVKOT bis(2-Aminoethyl)amine bis(3,5-dihydroxybenzoate); C4 H15 N3 2+,2(C7 H5 O4 1-

); C.Glidewell, G.Ferguson, R.M.Gregson, C.F.Campana; Acta Crystallogr.,Sect.B: Struct.Sci., 56, 68, 2000.

DEVFOZ bis(Pentafluorophenol) dioxane complex; C4 H8 O2,2(C6 H1 F5 O1); T.Gramstad, S.Husebye, K.Maartmann-Moe; Acta Chem.Scand.B, 39, 767, 1985.

DIPDIP10 2-(2-Amino-4-imidazolyl)ethylamine dipicrate monohydrate; C5 H12 N4 2+,2(C6 H2 N3 O7 1-),H2 O1; M.Nardelli, G.Pelizzi, F.Vitali, F.Bordi, P.V.Plazzi, T.Vitali; Acta Crystallogr.,Sect.C: Cryst.Struct.Commun., 43, 507, 1987.

DIXJEZ N N $\quad$ \$6!,N\$6!-Dimethyl-adeninium picrate; C7 H10 N5 1+,C6 H2 N3 O7 1-; T.Dahl; Acta Chem.Scand.B, 40, 226, 1986.

DOCRIW Triphenylphosphine oxide pentafluorophenol; C18 H15 O1 P1,C6 H1 F5 O1; T.Gramstad, S.Husebye, K.Maartmann-Moe; Acta Chem.Scand.B, 40, 26, 1986.

DOGWOL 2,5-Dimethyl-hydroquinone; C8 H10 O2; W.T.Pennington, A.O.Patil, I.C.Paul, D.Y.Curtin; J.Chem.Soc.,Perkin Trans.2, , 557, 1986.

DOGWOL01 2,5-Dimethyl-1,4-benzenediol; C8 H10 O2; G.J.Gainsford, I.J.Miller, S.C.Yorke, A.D.Rae; Acta Crystallogr.,Sect.C: Cryst.Struct.Commun., 53, 1673, 1997.

DOGWUR 2,3,5,6-Tetramethyl-hydroquinone; C10 H14 O2; W.T.Pennington, A.O.Patil, I.C.Paul, D.Y.Curtin; J.Chem.Soc.,Perkin Trans.2, , 557, 1986.

DOMQOL Dideutero-3-hydroxy-3-methoxy-1-azoniabicyclo(2.2.2)octane pentachlorophenolate; C8 H14 D2 N1 O2 1+,C6 Cl5 O1 1-; I.Majerz, Z.Malarski, T.Lis; Pol.J.Chem., 72, 325, 1998.

DOMQOL01 3-Hydroxy-3-methoxy-1-azoniabicyclo(2.2.2)octane pentachlorophenolate; C8 H16 N1 O2 1+,C6 Cl5 O1 1-; I.Majerz, Z.Malarski, T.Lis; Pol.J.Chem., 72, 325, 1998. DOTRUZ 2,3,4-Trihydroxyacetophenone; C8 H8 O4; E.O.Schlemper; Acta Crystallogr.,Sect.C: Cryst.Struct.Commun., 42, 755, 1986. 
DUGRUS bis(p-Cresol) N,N,N',N'-tetraisopropyloxamide; 2(C7 H8 O1),C14 H28 N2 O2;

F.Toda, Y.Tagami, T.C.W.Mak; Bull.Chem.Soc.Jpn., 59, 1189, 1986.

ECAYIQ 4-Amino-1-methylpyridinium p-hydroxybenzenesulfonate; C6 H9 N2 1+,C6 H5 O4

S1 1-; Anwar, S.Okada, H.Oikawa, H.Nakanishi; Chem.Mater., 12, 1162, 2000.

EDAFEU 2-Amino-5-nitropyridinium 4-chlorophenylsulfonate; C5 H6 N3 O2 1+,C6 H5 O4

S1 1-; H.Koshima, M.Hamada, I.Yagi, K.Uosaki; Cryst.Growth Des., 1, 467, 2001.

EFOMOB 1,3-dimethylurea picrate; C3 H9 N2 O1 1+,C6 H2 N3 O7 1-; R.Artali, G.Bombieri, C.Carvalho; Z.Kristallogr., 217, 88, 2002.

EGUTEF01 Ammonium 3,5-dinitrosalicylate; H4 N1 1+,C7 H3 N2 O7 1-; G.Smith,

U.D.Wermuth, R.C.Bott, P.C.Healy, J.M.White; Aust.J.Chem., 55, 349, 2002.

EGUTIJ Methylammonium 3,5-dinitrosalicylate; C1 H6 N1 1+,C7 H3 N2 O7 1-; G.Smith,

U.D.Wermuth, R.C.Bott, P.C.Healy, J.M.White; Aust.J.Chem., 55, 349, 2002.

EGUVAD Ethylenediammonium 3,5-dinitrosalicylate monohydrate; C2 H10 N2 2+,C7 H2 N2

O7 2-,H2 O1; G.Smith, U.D.Wermuth, R.C.Bott, P.C.Healy, J.M.White;

Aust.J.Chem., 55, 349, 2002.

EKENUD 2-Chloro-5-methylphenol; C7 H7 Cl1 O1; P.J.Cox; Acta Crystallogr.,Sect.C:

Cryst.Struct.Commun., 59, o533, 2003.

EKEPAL 4-Chloro-3-methylphenol; C7 H7 Cl1 O1; P.J.Cox; Acta Crystallogr.,Sect.C:

Cryst.Struct.Commun., 59, o533, 2003.

ELEWOH 4-Dimethylaminopyridinium 2,4-dinitrophenolate; C7 H1 1 N2 1+,C6 H3 N2 O5 1-;

N.Vembu, M.Nallu, E.C.Spencer, J.A.K.Howard; Acta Crystallogr.,Sect.E:

Struct.Rep.Online, 59, o1383, 2003.

ELIDUY 3,5-Di-t-butyl-2-hydroxybenzoic acid; C15 H22 O3; J.Mizuguchi; Acta

Crystallogr.,Sect.E: Struct.Rep.Online, 59, o1530, 2003. 
EMBIMP Ethyl N-(N'-methylbenzimidoyl)benzimidate picrate; C17 H19 N2 O1 1+,C6 H2 N3

O7 1-; P.G.Jones, O.Kennard; Acta Crystallogr.,Sect.B:

Struct.Crystallogr.Cryst.Chem., 33, 627, 1977.

FABZAJ10 4-Hydroxybiphenyl acetone solvate; C12 H10 O1,0.5(C3 H6 O1); C.P.Brock, G.L.Morelan; J.Phys.Chem., 90, 5631, 1986.

FADCES 2,3,5,6-Tetramethyl-1,4-benzoquinone 2,3,5,6-tetramethyl-1,4-hydroquinone; C10 H12 O2,C10 H14 O2; W.T.Pennington, A.O.Patil, D.Y.Curtin, I.C.Paul; J.Chem.Soc.,Perkin Trans.2, , 1693, 1986.

FADCIW 2,3,5,6-Tetramethyl-1,4-benzoquinone bis(hydroquinone); C10 H12 O2,2(C6 H6 O2); W.T.Pennington, A.O.Patil, D.Y.Curtin, I.C.Paul; J.Chem.Soc.,Perkin Trans.2, , 1693, 1986.

FARXOL N,N,N',N'-Tetracyclohexylfumaride bis(m-cresol) clathrate; C28 H46 N2 O2,2(C7 H8 O1); F.Toda, Y.Tagami, T.C.W.Mak; Chem.Lett., , 1909, 1986.

FAVXEF 3-(t-Butyldiazo)-2-(2-chlorobenzyl)-3-methyl-1,5,6,7,8,8a-hexahydro-(2H,3H)indolizinium picrate; C20 H31 Cl1 N3 1+,C6 H2 N3 O7 1-; M.H.Norman, C.H.Heathcock; J.Org.Chem., 52, 226, 1987.

FENXUR01 Hexamethyleneiminium p-hydroxybenzoate; C6 H14 N1 1+,C7 H5 O3 1-; Y.Moritani, N.Sasahara, S.Kashino, M.Haisa; Acta Crystallogr.,Sect.C: Cryst.Struct.Commun., 43, 154, 1987.

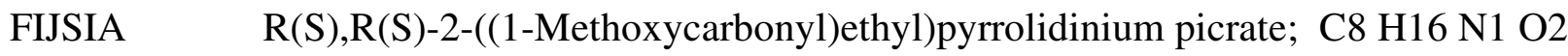
1+,C6 H2 N3 O7 1-; N.Knouzi, M.Vaultier, L.Toupet, R.Carrie; Tetrahedron Lett., 28, 1757, 1987.

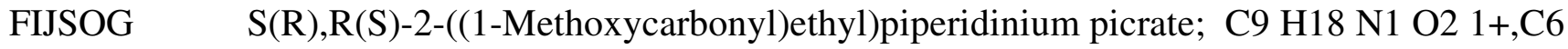
H2 N3 O7 1-; N.Knouzi, M.Vaultier, L.Toupet, R.Carrie; Tetrahedron Lett., 28, 1757, 1987. 
FOQHEY bis(phenazine) hydroquinone clathrate; 2(C12 H8 N2),C6 H6 O2; V.R.Thalladi, T.Smolka, R.Boese, R.Sustmann; CrystEngComm, , 17, 2000.

FUVNEP 4,4'-bis(Dimethylamino)benzophenone pentafluorophenol; C6 H1 F5 O1,C17 H20 N2 O1; T.Gramstad, S.Husebye, K.Maartmann-Moe, J.Saebo; Acta Chem.Scand.B, 41, 555, 1987.

FUXYEC bis(1,8-Epoxymenthane) 1,4-benzenediol; 2(C10 H18 O1),C6 H6 O2; J.C.Barnes; Acta Crystallogr.,Sect.C: Cryst.Struct.Commun., 44, 118, 1988.

GADGUN02 4-Methylpyridine pentachlorophenol; C6 H7 N1,C6 H1 Cl5 O1; Z.Malarski, I.Majerz, T.Lis; J.Mol.Struct., 380, 249, 1996.

GADGUN03 4-Methylpyridine pentachlorophenol; C6 H1 Cl5 O1,C6 H7 N1; T.Steiner, I.Majerz, C.C.Wilson; Angew.Chem.,Int.Ed.Engl., 40, 2651, 2001.

GADGUN04 4-Methylpyridine pentachlorophenol; C6 H1 Cl5 O1,C6 H7 N1; T.Steiner, I.Majerz, C.C.Wilson; Angew.Chem.,Int.Ed.Engl., 40, 2651, 2001.

GADGUN05 4-Methylpyridine pentachlorophenol; C6 H1 Cl5 O1,C6 H7 N1; T.Steiner, I.Majerz, C.C.Wilson; Angew.Chem.,Int.Ed.Engl., 40, 2651, 2001.

GAPWID bis(2-Methylphenol) 1,4-benzoquinone; C6 H4 O2,2(C7 H8 O1); K.Prout, J.Fail, R.M.Jones, R.E.Warner, J.C.Emmett; J.Chem.Soc.,Perkin Trans.2, , 265, 1988.

GEBMIJ Methoxycarbonylcholine picrate hemihydrate; C6 H2 N3 O7 1-,C7 H16 N1 O3 1+,0.5(H2 O1); K.Frydenvang, L.Gronborg, B.Jensen; Acta Crystallogr.,Sect.C: Cryst.Struct.Commun., 44, 841, 1988.

GEBMOP01 Ethoxycarbonylcholine picrate hemihydrate; C8 H18 N1 O3 1+,C6 H2 N3 O7 1,0.5(H2 O1); K.Frydenvang, P.Trickey, B.Jensen; Acta Crystallogr.,Sect.C: Cryst.Struct.Commun., 50, 1839, 1994.

GEBVAK 2-Amino-4-methylphenol; C7 H9 N1 O1; S.Kashino, M.Tomita, M.Haisa; Acta Crystallogr.,Sect.C: Cryst.Struct.Commun., 44, 730, 1988. 
GICKAE 1,2,3,4-Tetrahydro-1,4-dimethylisoquinolinium picrate; C11 H16 N1 1+,C6 H2 N3

O7 1-; W.H.Watson, A.Nagl, D.Minter, M.Re; Acta Crystallogr.,Sect.C:

Cryst.Struct.Commun., 44, 1681, 1988.

GIYJED anti-N,N'-bis(2-t-Butylphenyl)pyromellitic di-imide bis(catechol) clathrate; C30 H28 N2 O4,2(C6 H6 O2); K.Kishikawa, S.Tsubokura, S.Kohmoto, M.Yamamoto; J.Org.Chem., 64, 7568, 1999.

GOBYOL 1,4,10,13-Tetraoxa-7,16-diazacyclo-octadecane bis(p-nitrophenyl) clathrate; C12 H26 N2 O4,2(C6 H5 N1 O3); W.H.Watson, F.Vogtle, W.M.Muller; J.Inclusion Phenom.Macrocyclic Chem., 6, 491, 1988.

GOJNIC Morpholinium pentachlorophenolate monohydrate; C4 H10 N1 O1 1+,C6 Cl5 O1 1„H2 O1; I.Majerz, Z.Malarski, T.Lis; J.Mol.Struct., 161, 165, 1987.

GORSUB $\quad \mathrm{P}, \mathrm{P}-(1,4,8,12-$ Tetra-azacyclotetradecane-N,N')-N'-benzylidene-Nmethylthiophosphorhydrazone dipicrate; C18 H33 N6 P1 S1 2+,2(C6 H2 N3 O7 1-); D.Prevote, B.Donnadieu, M.Moreno-Manas, A.M.Caminade, J.P.Majoral; Eur.J.Org.Chem., , 1701, 1999.

GUFMAV 2,3,5,6-Tetrafluorohydroquinone; C6 H2 F4 O2; V.R.Thalladi, H.-C.Weiss, R.Boese, A.Nangia, G.R.Desiraju; Acta Crystallogr.,Sect.B: Struct.Sci., 55, 1005, 1999.

GUNPIC10 Guanine picrate monohydrate; C5 H6 N5 O1 1+,C6 H2 N3 O7 1-,H2 O1;

C.E.Bugg, U.Thewalt; Acta Crystallogr.,Sect.B: Struct.Crystallogr.Cryst.Chem., 31, $121,1975$.

GUSSES 1,3,5-tris(3-Dimethylamino-1-oxoprop-2-enyl)benzene hydroquinone; C21 H27 N3 O3,C6 H6 O2; A.-K.Pleier, E.Herdtweck, S.A.Mason, W.R.Thiel; Eur.J.Org.Chem., , 499, 2003. 
HABLIG 3-Hydroxyphenylammonium dihydrogen phosphate; C6 H8 N1 O1 1+,H2 O4 P1 1-; T.Glowiak, S.Debrus, M.May, A.J.Barnes, H.Ratajczak; J.Mol.Struct., 596, 77, 2001.

HABNON (+-)-(2S,4R,5S)-Tetrahydro-4-hydroxy-N,N,N,5-tetramethyl-2-furanaminium picrate;

C9 H20 N1 O2 1+,C6 H2 N3 O7 1-; K.Frydenvang, B.Jensen; Acta

Crystallogr.,Sect.C: Cryst.Struct.Commun., 49, 985, 1993.

HACBAO 2-Amino-1-hydroxy-5,5-dimethylpyrrolinium picrate; C6 H13 N2 O1 1+,C6 H2 N3

O7 1-; M.Hesse, B.R.Vincent, A.Linden; Private Communication, , , 1993.

HACTPH11 4-Hydroxyacetophenone; C8 H8 O2; A.J.Kresge, A.J.Lough, Yu Zhu; Acta

Crystallogr.,Sect.E: Struct.Rep.Online, 58, o1057, 2002.

HESKOF 2,5-Di-t-butylhydroquinone; C14 H22 O2; O.Ermer, C.Robke; Angew.Chem..Int.Ed.Engl., 33, 1755, 1994.

HEXGAS 4-Amino-3,5-dicyano-2,6-diphenylphenol; C20 H13 N3 O1; V.A.Tafeenko, T.V.Bogdan, L.A.Aslanov; Zh.Strukt.Khim.(Russ.)(J.Struct.Chem.), 35, 155-4, 1994.

HIBFED Piperazine bis(phenol); C4 H10 N2,2(C6 H6 O1); J.H.Loehlin, M.C.Etter, C.Gendreau, E.Cervasio; Chem.Mater., 6, 1218, 1994.

HIBFON Ethylenediamine bis(phenol); C2 H8 N2,2(C6 H6 O1); J.H.Loehlin, M.C.Etter, C.Gendreau, E.Cervasio; Chem.Mater., 6, 1218, 1994.

HIBGII 2,7-Dimethyltricyclo(4.3.1.1\$3,8!)undecane-syn-2,syn-7-diol p-chlorophenol; C13 H22 O2,C6 H5 Cl1 O1; A.T.Ung, R.Bishop, D.C.Craig, I.G.Dance, M.L.Scudder; Chem.Mater., 6, 1269, 1994.

HIBGUU 2,7-Dimethyltricyclo(4.3.1.1\$3,8!)undecane-syn-2,syn-7-diol p-hydroxythiophenol clathrate; C13 H22 O2,C6 H6 O1 S1; A.T.Ung, R.Bishop, D.C.Craig, I.G.Dance, M.L.Scudder; Chem.Mater., 6, 1269, 1994. 
HIBHAB 2,8-Dimethyltricyclo(5.3.1.1\$3,9!)dodecane-syn-2,syn-8-diol phenol clathrate; C14 H24 O2,C6 H6 O1; A.T.Ung, R.Bishop, D.C.Craig, I.G.Dance, M.L.Scudder; Chem.Mater., 6, 1269, 1994.

HIBHEF bis(2,8-Dimethyltricyclo(5.3.1.1\$3,9!)dodecane-syn-2,syn-8-diol) phloroglucinol clathrate; 2(C14 H24 O2),C6 H6 O3; A.T.Ung, R.Bishop, D.C.Craig, I.G.Dance, M.L.Scudder; Chem.Mater., 6, 1269, 1994.

HICCOL Decahydro-6,6-dimethoxy-1,2,2,4-tetramethylquinolinium picrate; C15 H30 N1 O2 1+,C6 H2 N3 O7 1-; O.Kasemann, P.Stanetty, K.Mereiter; Acta Crystallogr.,Sect.C: Cryst.Struct.Commun., 51, 306, 1995.

HIFWIC hexakis(p-Phenolammonium) cyclo-hexaphosphate octahydrate; 6(C6 H8 N1 O1 1+),O18 P6 6-,8(H2 O1); E.H.Soumhi, T.Jouini; Acta Crystallogr.,Sect.C: Cryst.Struct.Commun., 52, 434, 1996.

HIHNER 1,4,10-Trioxa-7,13-diazacyclopentadecane dipicric acid clathrate; C10 H24 N2 O3 2+,2(C6 H2 N3 O7 1-); M.I.Saleh, A.Salhin, B.Saad, K.Sivakumar, Hoong-Kun Fun; Acta Crystallogr.,Sect.C: Cryst.Struct.Commun., 52, 1509, 1996.

HIKWAZ 1,3-Di-t-butyl-5-methyltetrazolium picrate; C10 H21 N4 1+,C6 H2 N3 O7 1-;

P.N.Gaponik, S.V.Voitekhovich, O.A.Ivashkevich, A.S.Lyakhov, A.A.Govorova;

Khim.Get.Soedin.,SSSR(Russ.)(Chem.Hetero.Compnd), , 657, 1998.

HIMGAL 1,3,5-Trihydroxybenzene tris(4-methylpyridine); C6 H6 O3,3(C6 H7 N1);

K.Biradha, M.J.Zaworotko; J.Am.Chem.Soc., 120, 6431, 1998.

HIMGEP 1,3,5-Trihydroxybenzene tris(2,4-dimethylpyridine); C6 H6 O3,3(C7 H9 N1);

K.Biradha, M.J.Zaworotko; J.Am.Chem.Soc., 120, 6431, 1998.

HMTHQU Hexamethylenetetramine-hydroquinone; C6 H12 N4,C6 H6 O2; T.C.W.Mak, C.-

S.Tse, Y.-H.Chong, F.-C.Mok; Acta Crystallogr.,Sect.B:

Struct.Crystallogr.Cryst.Chem., 33, 2980, 1977. 
HQUPRD Hydroquinone 1,1'-dimethyl-4,4'-bipyridylium dichloride tetrahydrate; C6 H6 O2,C12 H14 N2 2+,2(C11 1-),4(H2 O1); M.M.Mahmoud, S.C.Wallwork; Acta Crystallogr.,Sect.B: Struct.Crystallogr.Cryst.Chem., 37, 398, 1981.

HUZDOV Tetra-n-butylammonium 2,6-dihydroxybenzoate 2,6-dihydroxybenzoic acid solvate; C16 H36 N1 1+,C7 H5 O4 1-,C7 H6 O4; F.A.A.Paz, P.C.R.Soares-Santos, H.I.S.Nogueira, T.Trindade, J.Klinowski; Acta Crystallogr.,Sect.E:

Struct.Rep.Online, 59, o506, 2003.

HUZSIE 2-Aminobenzimidazolium picrate; C7 H8 N3 1+,C6 H2 N3 O7 1-; S.M.El-Medani, T.A.Youssef, R.M.Ramadan; J.Mol.Struct., 644, 77, 2003.

HYQUIN05 Hydroquinone; C6 H6 O2; S.V.Lindeman, V.E.Shklover, Yu.T.Struchkov; Cryst.Struct.Commun., 10, 1173, 1981.

HYQUIN06 Hydroquinone; C6 H6 O2; M.Bolte, H.-W.Lerner; Private Communication, , , 2001.

IDUMOJ bis(N,N-Diethyl)bicyclo[2.2.2]octane-1,4-dicarboxamide hydroquinone; C18 H32 N2 O2,C6 H6 O2; B.M.Foxman, D.J.Guarrera, R.Pai, C.Tassa, J.C.Warner; Crystal Engineering, 2, 55, 1999.

IDUMUP $\quad$ bis[bis(N,N-Dimethyl)bicyclo[2.2.2]octane-1,4-dicarboxamide] hydroquinone; 2(C14 H24 N2 O2),C6 H6 O2; B.M.Foxman, D.J.Guarrera, R.Pai, C.Tassa, J.C.Warner; Crystal Engineering, 2, 55, 1999.

IDUNAW bis(N,N-Dimethyl)bicyclo[2.2.2] $\quad$ octane-1,4-dicarboxamide bis(hydroquinone); C14 H24 N2 O2,2(C6 H6 O2); B.M.Foxman, D.J.Guarrera, R.Pai, C.Tassa, J.C.Warner; Crystal Engineering, 2, 55, 1999.

IHERIW Hexamethylenetetraamine 4-nitrocatechol monohydrate; C6 H12 N4,2(C6 H5 N1 O4),H2 O1; S.Chantrapromma, A.Usman, Hoong-Kun Fun, Bo-Long Poh, C.Karalai; Acta Crystallogr.,Sect.C: Cryst.Struct.Commun., 58, o675, 2002. 
IJIYOP 4-Methylpyridinium 2,4-dinitrophenolate; C6 H3 N2 O5 1-,C6 H8 N1 1+; Zhi Min Jin, Ji Zhong Yan, Ya Ping Lu, Ling He, Nan Sun; J.Chem.Cryst., 31, 345, 2001.

IJUPEI $\quad \operatorname{rac}-\left(6 a R^{*}, 10 a S^{*}, 10 \mathrm{bR} *\right)-2-M e t h y l-10 a-h y d r o x y-3,4,6,6 a, 7,8,9,10,10 \mathrm{a}, 10 \mathrm{~b}-$ decahydrocyclohexa(a)indolizin-5-ium picrate; C13 H22 N1 O1 1+,C6 H2 N3 O7 1; J.Heimann, H.J.Schafer, R.Frohlich, B.Wibbeling; Eur.J.Org.Chem., , 2919, 2003.

IPRPOL01 4-Isopropylphenol; C9 H12 O1; G.Wojcik, J.Holband; Acta Crystallogr.,Sect.B: Struct.Sci., 58, 684, 2002.

IPRPOL02 4-Isopropylphenol; C9 H12 O1; G.Wojcik, J.Holband; Acta Crystallogr.,Sect.B: Struct.Sci., 58, 684, 2002.

IPRPOL03 4-Isopropylphenol; C9 H12 O1; G.Wojcik, J.Holband; Acta Crystallogr.,Sect.B: Struct.Sci., 58, 684, 2002.

JIXCOI p-Hydroxybenzamide monohydrate; C7 H7 N1 O2,H2 O1; S.Kashino, S.Tateno, H.Tanabe, M.Haisa, Y.Katsube; Acta Crystallogr.,Sect.C: Cryst.Struct.Commun., 47, 2236, 1991.

JIZZIB 4-Methoxy-2,6-dimethylpyridinium N-hydroxide 2,6-dichloro-4-nitrophenolate; C8 H12 N1 O2 1+,C6 H2 Cl2 N1 O3 1-; Z.Dega-Szafran, A.Kania, M.GrundwaldWyspianska, M.Szafran, E.Tykarska; J.Mol.Struct., 381, 107, 1996.

JOYQUJ01 2,5-Dibromohydroquinone; C6 H4 Br2 O2; M.Bolte, G.Margraf; Private Communication, , , 2002.

JOZZIH p-Hydroxybenzoic acid; C7 H6 O3; E.A.Heath, P.Singh, Y.Ebisuzaki; Acta Crystallogr.,Sect.C: Cryst.Struct.Commun., 48, 1960, 1992.

JOZZON bis(p-Hydroxybenzoic acid) acetone solvate; 2(C7 H6 O3),C3 H6 O1; E.A.Heath, P.Singh, Y.Ebisuzaki; Acta Crystallogr.,Sect.C: Cryst.Struct.Commun., 48, 1960, 1992. 
JUDNAX 4-Nitropyridine N-oxide 4-nitrophenol; C5 H4 N2 O3,C6 H5 N1 O3; R.Moreno

Fuquen, R.H.de Almeida Santos, J.R.Lechat; J.Crystallogr.Spectrosc.Res., 22, 201, 1992.

JUDVIN Hydroquinone bis(N,N-diethyl)terephthalamide; C6 H6 O2,C16 H24 N2 O2;

B.M.Foxman, D.J.Guarrera, L.D.Taylor, D.VanEngen, J.C.Warner; Crystal Engineering, 1, 109, 1998.

JUSRUK Isoquinolinium picrate; C9 H8 N1 1+,C6 H2 N3 O7 1-; M.Goto, H.Takayanagi,

K.Furuhata, H.Ogura, K.Saito, K.Sugai, N.Sugiyama; Chem.Pharm.Bull., 40, 1612, 1992.

JUVBOR Sodium 4-hydroxy-2,3,5,6-tetracyanophenoxide; C10 H1 N4 O2 1-,Na1 1+;

C.Vazquez, J.C.Calabrese, D.A.Dixon, J.S.Miller; J.Org.Chem., 58, 65, 1993.

JUVCAE Tetra-n-butylammonium 4-hydroxy-2,3,5,6-tetracyanophenoxide; C10 H1 N4 O2 1,C16 H36 N1 1+; C.Vazquez, J.C.Calabrese, D.A.Dixon, J.S.Miller; J.Org.Chem., $58,65,1993$.

JUVCOS Tetramethylammonium 4-hydroxy-2,3,5,6-tetracyanophenoxide; C10 H1 N4 O2 1-

,C4 H12 N1 1+; C.Vazquez, J.C.Calabrese, D.A.Dixon, J.S.Miller; J.Org.Chem., $58,65,1993$.

KABSIP tetrakis(p-Phenolammonium) cyclotetraphosphate hexahydrate; 4(C6 H8 N1 O1 1+),O12 P4 4-,6(H2 O1); E.H.Soumhi, I.Saadoune, A.Driss, T.Jouini; J.Solid State Chem., 144, 318, 1999.

KATCEN Ammonium 2-chloro-4,6-dinitrophenolate sesquihydrate; C6 H2 Cl1 N2 O5 1-,H4

N1 1+,1.5(H2 O1); E.K.Andersen, I.G.K.Andersen, G.Ploug-Sorensen; Acta Chem.Scand., 43, 624, 1989.

KOCJOB N-Benzyl-2-(p-tolylsulfinyl)propylamine picrate; C17 H22 N1 O1 S1 1+,C6 H2 N3

O7 1-; R.Kawecki, L.Kozerski, Z.Urbanczyk-Lipkowska, G.Bocelli;

J.Chem.Soc.,Perkin Trans.1, , 2255, 1991. 
KONTIQ 3,4,5-Trihydroxybenzoic acid monohydrate; C7 H6 O5,H2 O1; Ren-Wang Jiang, Dong-Sheng Ming, P.P.H.But, T.C.W.Mak; Acta Crystallogr.,Sect.C:

Cryst.Struct.Commun., 56, 594, 2000.

KONTIQ01 3,4,5-Trihydroxybenzoic acid monohydrate; C7 H6 O5,H2 O1; N.Okabe,

H.Kyoyama, M.Suzuki; Acta Crystallogr.,Sect.E: Struct.Rep.Online, 57, o764, 2001.

KOYWAW Choline picrate; C5 H14 N1 O1 1+,C6 H2 N3 O7 1-; K.Frydenvang, B.Jensen,

K.Nielsen; Acta Crystallogr.,Sect.C: Cryst.Struct.Commun., 48, 1343, 1992.

LEQVOS Betaine 2,6-dichloro-4-nitrophenol; C5 H12 N1 O2 1+,C6 H2 Cl2 N1 O3 1-;

Z.Dega-Szafran, A.Komasa, M.Grundwald-Wyspianska, M.Szafran, G.Buczak,

A.Katrusiak; J.Mol.Struct., 404, 13, 1997.

LEWPOS 2-Aminopyrimidinium 2,6-dihydroxybenzoate; C4 H6 N3 1+,C7 H5 O4 1-;

D.E.Lynch, G.Smith, D.Freney, K.A.Byriel, C.H.L.Kennard; Aust.J.Chem., 47, 1097, 1994.

LEWRUA 2-Aminopyridinium 2,6-dihydroxybenzoate; C5 H7 N2 1+,C7 H5 O4 1-;

D.E.Lynch, G.Smith, D.Freney, K.A.Byriel, C.H.L.Kennard; Aust.J.Chem., 47, 1097, 1994.

LEZJAB 2,6-Dihydroxybenzoic acid; C7 H6 O4; M.Gdaniec, M.Gilski, G.S.Denisov; Acta Crystallogr.,Sect.C: Cryst.Struct.Commun., 50, 1622, 1994.

LEZJEF 2,6-Dihydroxybenzoic acid monohydrate; C7 H6 O4,H2 O1; M.Gdaniec, M.Gilski, G.S.Denisov; Acta Crystallogr.,Sect.C: Cryst.Struct.Commun., 50, 1622, 1994.

LOLSUA 2-Methylpyridine pentachlorophenol; C6 H7 N1,C6 H1 Cl5 O1; T.Steiner, C.C.Wilson, I.Majerz; Chem.Commun., , 1231, 2000.

LUNMEM Isonicotinamide 3-hydroxybenzoic acid; C6 H6 N2 O1,C7 H6 O3; C.B.Aakeroy, A.M.Beatty, B.A.Helfrich; J.Am.Chem.Soc., 124, 14425, 2002. 
LUPCAA bis(1,1'-Dimethyl-4,4'-bipyridinium) hydroquinone bis(anthraquinone-2,7disulfonate); 2(C12 H14 N2 2+),C6 H6 O2,2(C14 H6 O8 S2 2-); M.Kidowaki, N.Tamaoki; Chem.Commun., , 290, 2003.

MAMPOL02 3-Aminophenol; C6 H7 N1 O1; F.H.Allen, V.J.Hoy, J.A.K.Howard, V.R.Thalladi, G.R.Desiraju, C.C.Wilson, G.J.McIntyre; J.Am.Chem.Soc., 119, 3477, 1997.

MANMUJ Betaine bis(imidazole picric acid); C5 H11 N1 O2,2(C3 H5 N2 1+),2(C6 H2 N3 O7 1-); J.Overgaard, B.Schiott, F.K.Larsen, A.J.Schultz, J.C.MacDonald, B.B.Iversen; Angew.Chem.,Int.Ed.Engl., 38, 1239, 1999.

MASBUD 3,4-Dihydroxybenzaldehyde; C7 H6 O3; J.A.R.P.Sarma, A.Nagaraju, K.K.Majumdar, P.M.Samuel, I.Das, S.Roy, A.J.McGhie; J.Chem.Soc.,Perkin Trans.2, , 1119, 2000.

MCHTEP03 Dimethyl 3,6-dichloro-2,5-dihydroxyterephthalate; C10 H8 Cl2 O6; Qing-Chuan Yang, M.F.Richardson, J.D.Dunitz; Acta Crystallogr.,Sect.B: Struct.Sci., 45, 312, 1989.

MCHTEP04 Dimethyl 3,6-dichloro-2,5-dihydroxyterephthalate; C10 H8 Cl2 O6; Qing-Chuan Yang, M.F.Richardson, J.D.Dunitz; Acta Crystallogr.,Sect.B: Struct.Sci., 45, 312, 1989.

MCHTEP05 Dimethyl 3,6-dichloro-2,5-dihydroxyterephthalate; C10 H8 Cl2 O6; Qing-Chuan Yang, M.F.Richardson, J.D.Dunitz; Acta Crystallogr.,Sect.B: Struct.Sci., 45, 312, 1989.

MEKWUU 1,2-bis(4-Pyridyl)ethane p-resorcinol; C12 H12 N2,C6 H6 O2; E.Corradi, S.V.Meille, M.T.Messina, P.Metrangolo, G.Resnati; Angew.Chem.,Int.Ed.Engl., 39, $1782,2000$.

MEVXIU 3,5,7-triaza-1-azoniatricyclo(3.3.1.1\$3,7!)decane 2,4-dinitrophenolate monohydrate; C6 H13 N4 1+,C6 H3 N2 O5 1-,H2 O1; A.Usman, S.Chantrapromma, Hoong-Kun Fun; Acta Crystallogr.,Sect.C: Cryst.Struct.Commun., 57, 1443, 2001. 
MEVXIU01 3,5,7-triaza-1-azoniatricyclo(3.3.1.1\$3,7!)decane 2,4-dinitrophenolate monohydrate; C6 H13 N4 1+,C6 H3 N2 O5 1-,H2 O1; A.Usman, S.Chantrapromma, Hoong-Kun Fun; Acta Crystallogr.,Sect.C: Cryst.Struct.Commun., 57, 1443, 2001

MIFQAT 1,4-Diazabicyclo(2.2.2)octanium 2,4-dinitrophenolate; C6 H13 N2 1+,C6 H3 N2 O5

1-; S.Chantrapromma, A.Usman, H.-K.Fun, Bo-Long Poh, C.Karalai; Acta Crystallogr.,Sect.E: Struct.Rep.Online, 58, o102, 2002.

MIFQIB N,N-Dimethylethylenediamine-1,4-diium 2,4-dinitrophenolate; C4 H14 N2 2+,2(C6 H3 N2 O5 1-); S.Chantrapromma, A.Usman, H.-K.Fun, Bo-Long Poh, C.Karalai; Acta Crystallogr.,Sect.E: Struct.Rep.Online, 58, o108, 2002.

MILLIC 1,3,5,7-Tetra-aza-adamantan-1-ium 2-carboxy-4,6-dinitrophenolate; C6 H13 N4 1+,C7 H3 N2 O7 1-; S.W.Ng, P.Naumov, M.G.B.Drew, G.Wojciechowskid, B.Brzezinski; J.Mol.Struct., 595, 29, 2001.

MILLIC01 Hexamethylenetetramin-1-ium 3,5-dinitrosalicylate; C6 H13 N4 1+,C7 H3 N2 O7 1; G.Smith, U.D.Wermuth, R.C.Bott, P.C.Healy, J.M.White; Aust.J.Chem., 55, 349, 2002.

MILLUO 1,5,7-Triazabicyclo[4.4.0]dec-5-enium 2-hydroxy-4-nitrophenoxide; C7 H14 N3 1+,C6 H4 N1 O4 1-; S.W.Ng, P.Naumov, S.Chantrapromma, S.S.S.Raj, H.-K.Fun, A.R.Ibrahim, G.Wojciechowski, B.Brzezinski; J.Mol.Struct., 569, 139, 2001.

MIPROS 8-Aminoquinolinium 3,5-dinitrosalicylate; C9 H9 N2 1+,C7 H3 N2 O7 1-; G.Smith, U.D.Wermuth, R.C.Bott, J.M.White, A.C.Willis; Aust.J.Chem., 54, 165, 2001.

MIRBAQ 2-Methyl-6-(2-methylpropyl)piperidinium picrate; C10 H22 N1 1+,C6 H2 N3 O7 1-; G.A.Molander, E.D.Dowdy, S.K.Pack; J.Org.Chem., 66, 4344, 2001.

MIYLIP 2-(4-Hydroxyphenyl)ethylammonium picrate; C6 H2 N3 O7 1-,C8 H12 N1 O1 1+;

S.Ohba, Y.Ito; Acta Crystallogr.,Sect.E: Struct.Rep.Online, 58, o584, 2002.

MNPHOL02 m-Nitrophenol; C6 H5 N1 O3; F.Pandarese, L.Ungaretti, A.Coda; Acta Crystallogr.,Sect.B: Struct.Crystallogr.Cryst.Chem., 31, 2671, 1975. 
MNPHOL10 m-Nitrophenol; C6 H5 N1 O3; F.Pandarese, L.Ungaretti, A.Coda; Acta

Crystallogr.,Sect.B: Struct.Crystallogr.Cryst.Chem., 31, 2671, 1975.

MOXBUW Hydrogen semicarbazide picrate; C1 H6 N3 O1 1+,C6 H2 N3 O7 1-; Li Yang,

Tong-Lai Zhang, Jian-Guo Zhang, Bing Shao, Chang-Gen Feng, Kai-Bei Yu;

Hanneng Cailiao Nagasaki Daigaku(Chin.)(Energet.Mater.), 9, 73, 2001.

MUFQOT 9,10-Diallyl-11-methyl-9-azoniatricyclo(6.2.2.0\$2,7!)dodeca-2(7),3,5-triene picrate;

C18 H24 N1 1+,C6 H2 N3 O7 1-; Yu.N.Bubnov, F.M.Pastukhov, Z.A.Starikova, A.V.Ignatenko; Izv.Akad.Nauk SSSR,Ser.Khim.(Russ.)(Russ.Chem.Bull.), , 2074, 2001.

MUFRAG 11,11-Dimethyl-10-(2-methylprop-2-enyl)-9-azoniatricyclo(6.2.2.0\$2,7!)dodeca2(7),3,5-triene picrate methanol solvate; C17 H24 N1 1+,C6 H2 N3 O7 1-,C1 H4

O1; Yu.N.Bubnov, F.V.Pastukhov, Z.A.Starikova, A.V.Ignatenko; Izv.Akad.Nauk SSSR,Ser.Khim.(Russ.)(Russ.Chem.Bull.), , 2074, 2001.

MUHRAI Dodecylammonium picrate; C12 H28 N1 1+,C6 H2 N3 O7 1-; V.Tomasic, Lj.Tusek-Bozik, A.Visnjevac, B.Kojic-Prodic, N.Filipovic-Vincekovic; J.Colloid Interface Sci., 227, 427, 2000.

MUKJUX 1,4,5,6-Tetramethyl-1,2,3,4,5,6-hexahydronaphtho(2,3-e)(1,4)diazocin-4-ium 2,4,6trinitrophenolate; C18 H25 N2 1+,C6 H2 N3 O7 1-; W.Rubin, M.Heuschmann, K.Polborn; Private Communication, , , 2002.

NABMAE Aminopicric acid; C6 H4 N4 O7; H.Adolf, A.L.Rheingold, M.B.Allen; Private Communication, , , 1996.

NECFUW 2,5-bis(Trimethylsilyl)hydroquinone; C12 H22 O2 Si2; H.Bock, S.Nick, C.Nather, J.W.Bats; J.Prakt.Chem.-Chem.-Zeitung, 338, 363, 1996.

NECGAD 2,5-bis(Trimethylsilyl)hydroquinone dimethoxyethane solvate; 3(C12 H22 O2 Si2),2(C4 H10 O2); H.Bock, S.Nick, C.Nather, J.W.Bats; J.Prakt.Chem.-Chem.Zeitung, 338, 363, 1996. 
NECGEH 2,5-bis(Trimethylsilyl)-1,6-dihydroxybenzene dioxane solvate; C12 H22 O2

Si2,2(C4 H8 O2); H.Bock, S.Nick, C.Nather, J.W.Bats; J.Prakt.Chem.-Chem.-

Zeitung, 338, 363, 1996.

NEJHEP 5,6-Butano-2-hexyl-7-phenylthio-9-propenyl-1-azoniabicyclo(4.3.0)nonane picrate;

C27 H42 N1 S1 1+,C6 H2 N3 O7 1-; W.H.Pearson, N.S.Barta, J.W.Kampf;

Tetrahedron Lett., 38, 3369, 1997.

NEJHIT 5,6-Butano-2-hexyl-7-phenylsulfinyl-9-hydroxymethyl-1-

azoniabicyclo(4.3.0)nonane picrate; C25 H40 N1 O2 S1 1+,C6 H2 N3 O7 1-;

W.H.Pearson, N.S.Barta, J.W.Kampf; Tetrahedron Lett., 38, 3369, 1997.

NELTIH 2,4-Dinitrophenol 4-methoxypyridine N-oxide; C6 H4 N2 O5,C6 H7 N1 O2;

R.Moreno-Fuquen, F.Cano, M.Martinez-Ripoll, A.Montano, J.Zukerman-Schpector; Acta Crystallogr.,Sect.E: Struct.Rep.Online, 57, o712, 2001.

NEMTAA 5-Nitrosalicyclic acid phenylurea; C7 H5 N1 O5,C7 H8 N2 O1; R.C.Bott, G.Smith, U.D.Wermuth, N.C.Dwyer; Aust.J.Chem., 53, 767, 2000.

NILZOX Pyridine N-oxide 4-nitrophenol; C6 H5 N1 O3,C5 H5 N1 O1; R.Moreno-Fuquen,

R.H.De Almeida Santos, R.H.P.Francisco; Acta Crystallogr.,Sect.C:

Cryst.Struct.Commun., 54, 513, 1998.

NILZUD bis(3-Picoline N-oxide) hydroquinone; C6 H6 O2,2(C6 H7 N1 O1); R.Moreno-

Fuquen, M.T.do Prada Gambardella, J.Valderrama-N; Acta Crystallogr.,Sect.C:

Cryst.Struct.Commun., 54, 515, 1998.

NIMDIW 8-Hydroxyquinoline salicylate salicylic acid; C9 H8 N1 O1 1+,C7 H5 O3 1-,C7 H6

O3; J.R.Jebamony, P.T.Muthiah; Acta Crystallogr.,Sect.C: Cryst.Struct.Commun., 54, 539, 1998.

NINSOS Morpholinium 2,4-dinitrophenolate; C4 H10 N1 O1 1+,C6 H3 N2 O5 1-; I.Majerz, T.Glowiak, A.Koll; J.Mol.Struct., 374, 339, 1996. 
NITPOL02 4-Nitrophenol; C6 H5 N1 O3; G.U.Kulkarni, P.Kumaradhas, C.N.R.Rao; Chem.Mater., 10, 3498, 1998.

NITPOL03 4-Nitrophenol; C6 H5 N1 O3; G.U.Kulkarni, P.Kumaradhas, C.N.R.Rao;

Chem.Mater., 10, 3498, 1998.

NIXKIO Dibenzylideneacetone picric acid; C6 H3 N3 O7,C17 H14 O1; K.Biradha, A.Nangia, G.R.Desiraju, C.J.Carrell, H.L.Carrell; J.Mater.Chem., 7, 1111, 1997.

NODTUV 2-Amino-4-nitrophenol methanol solvate; C6 H6 N2 O3,C1 H4 O1; N.Blagden, W.I.Cross, R.J.Davey, M.Broderick, R.G.Pritchard, R.J.Roberts, R.C.Rowe; Phys.Chem.Chem.Phys.(PCCP), 3, 3819, 2001.

NOPSFC N-(p-Chlorophenyl)-S,S-dimethylsulfimide picrate; C6 H2 N3 O7 1-,C8 H1 1 Cl1

N1 S1 1+; A.F.Cameron, A.A.Freer, A.Maltz; Acta Crystallogr.,Sect.B:

Struct.Crystallogr.Cryst.Chem., 37, 1608, 1981.

NPOAPL 4-Nitropyridine-N-oxide 3-aminophenol; C5 H4 N2 O3,C6 H7 N1 O1; J.R.Lechat,

R.H.de Almeida Santos, W.A.Bueno; Acta Crystallogr.,Sect.B:

Struct.Crystallogr.Cryst.Chem., 37, 1468, 1981.

NTSALA 5-Nitrososalicylic acid; C7 H5 N1 O4; H.J.Talberg; Acta Chem.Scand.A, 31, 485, 1977.

NUJDOL N,N-bis(Benzylidene)ethylenediamine hydroquinone; C16 H16 N2,C6 H6 O2;

K.Panneerselvam, M.Soriano-Garcia, A.Reyes-Arellano, J.Tamariz-Mascarua, R.I.Mendoza-Sanchez; Anal.Sci., 12, 823, 1996.

NUKBIE 4-(Dimethylamino)pyridinium 2,6-dichloro-4-nitrophenolate; C7 H11 N2 1+,C6 H2 Cl2 N1 O3 1-; I.Majerz, W.Sawka-Dobrowolska, L.Sobczyk; J.Mol.Struct., 375, 37, 1996.

OCAYOG Melaminium bis(4-hydroxybenzenesulfonate) dihydrate; C3 H8 N6 2+,2(C6 H5 O4 S1 1-),2(H2 O1); J.Janczak, G.J.Perpetuo; Acta Crystallogr.,Sect.C: Cryst.Struct.Commun., 57, 873, 2001. 
OFECAD 1,5,7-Triazabicyclo(4.4.0)dec-5-ene bis(4-nitrophenol); C7 H14 N3 1+,C6 H4 N1

O3 1-,C6 H5 N1 O3; S.W.Ng, P.Naumov, S.Chantrapromma, S.S.S.Raj, H.-K.Fun, A.B.Ibrahim, G.Wojciechowski, B.Brzezinski; J.Mol.Struct., 562, 185, 2001.

OFUGUR 2-Pyridone 4-nitrophenol; C5 H5 N1 O1,C6 H5 N1 O3; I.Takahashi, M.Takahashi, T.Morita, T.Keumi, H.Kitajima, M.Wagi, M.Hatanaka, T.Ohta, S.Hosoi; Anal.Sci., 18, 619, 2002.

OFUHIG Hydroquinone bis(2-aminopyrimidine); C6 H6 O2,2(C4 H5 N3); Ning Shan, A.D.Bond, W.Jones; Tetrahedron Lett., 43, 3101, 2002.

OGAGUY bis(5-Nitrosalicylic acid) trans-1,4-dithiane-1,4-dioxide; 2(C7 H5 N1 O5),C4 H8 O2 S2; V.S.S.Kumar, A.Nangia, A.K.Katz, H.L.Carrell; Cryst.Growth Des., 2, 313, 2002.

OHBZSF10 Diethylammonium 2,5-dihydroxybenzene-1,4-disulfonate; C6 H4 O8 S2 2-,2(C4 H12 N1 1+); X.Solans, F.Plana, M.Font-Altaba; Acta Crystallogr.,Sect.B: Struct.Crystallogr.Cryst.Chem., 38, 651, 1982.

OKIRAB (R,R)-(-)-trans-1,2-Diaminocyclohexane benzene-1,4-diol; C6 H14 N2,C6 H6 O2; K.Tanaka, M.Kato, F.Toda; Chirality, 13, 347, 2001.

PABBAV 4-Acetylpyridine pentachlorophenol; C7 H7 N1 O1,C6 H1 Cl5 O1; I.Majerz, Z.Malarski, W.Sawka-Dobrowolska; J.Mol.Struct., 249, 109, 1991.

PABBEZ Acridinium pentachlorophenolate; C13 H10 N1 1+,C6 Cl5 O1 1-; K.Wozniak, T.M.Krygowski, B.Kariuki, W.Jones; J.Mol.Struct., 248, 331, 1991.

PAMZOS Morpholinium 2,3-dicyanohydroquinone; C4 H10 N1 O1 1+,C8 H3 N2 O2 1-; H.Schodel, W.Seitz, H.Bock, J.W.Bats; Monatsh.Chem., 127, 63, 1996.

PARMIE (1-Dimethylamino-8-dimethylammonium)naphthalene pentafluorophenoxide bis(pentafluorophenol); C14 H19 N2 1+,C6 F5 O1 1-,2(C6 H1 F5 O1); N.Odiaga, J.A.Kanters, B.T.G.Lutz, E.Grech; J.Mol.Struct., 273, 183, 1992. 
PARNAX beta-2,4,6-Trimethylpyridinium pentachlorophenolate; C8 H12 N1 1+,C6 Cl5 O1 1-

; I.Majerz, Z.Malarski, W.Sawka-Dobrowolska; J.Mol.Struct., 273, 161, 1992.

PECBII 1,4-Dihydroxy-2,3,5,6-tetracyanobenzene; C10 H2 N4 O2; H.Bock, W.Seitz, Z.Havlas, J.W.Bats; Angew.Chem.,Int.Ed.Engl., 32, 411, 1993.

PEZLOV N-Aminobenzimidazolium picrate; C7 H8 N3 1+,C6 H2 N3 O7 1-; R.M.Claramunt, D.Sanz, J.Catalan, F.Fabero, N.A.Garcia, C.Foces-Foces, A.L.Llamas-Saiz, J.Elguero; J.Chem.Soc.,Perkin Trans.2, , 1687, 1993.

PEZLOV01 N-Aminobenzimidazolium picrate; C7 H8 N3 1+,C6 H2 N3 O7 1-; R.M.Claramunt, D.Sanz, J.Catalan, F.Fabero, N.A.Garcia, C.Foces-Foces, A.L.Llamas-Saiz, J.Elguero; J.Chem.Soc.,Perkin Trans.2, , 1687, 1993.

PEZXIB 3-Methylpyridinium 2,6-dichloro-4-nitrophenoxide; C6 H2 Cl2 N1 O3 1-,C6 H8 N1 1+; I.Majerz, W.Sawka-Dobrowolska, L.Sobczyk; J.Mol.Struct., 297, 177, 1993.

PHBALD10 p-Hydroxybenzaldehyde; C7 H6 O2; F.Iwasaki; Acta Crystallogr.,Sect.B: Struct.Crystallogr.Cryst.Chem., 33, 1646, 1977.

PHBZAC01 p-Hydroxybenzoic acid monohydrate; C7 H6 O3,H2 O1; M.Colapietro, A.Domenicano, C.Marciante; Acta Crystallogr.,Sect.B:

Struct.Crystallogr.Cryst.Chem., 35, 2177, 1979.

PHENOL03 Phenol; C6 H6 O1; V.E.Zavodnik, V.K.Bel'skii, P.M.Zorkii; Zh.Strukt.Khim.(Russ.)(J.Struct.Chem.), 28, 175-5, 1987.

PHENOL11 Phenol; C6 H6 O1; D.R.Allan, S.J.Clark, A.Dawson, P.A.McGregor, S.Parsons; Acta Crystallogr.,Sect.B: Struct.Sci., 58, 1018, 2002.

POCVUY 3,4-Dimethylpyridinium 2,6-dichloro-4-nitrophenolate; C7 H10 N1 1+,C6 H2 Cl2 N1 O3 1-; I.Majerz, W.Sawka-Dobrowolska, L.Sobczyk; J.Mol.Struct., 319, 1, 1994. 
POCWAF02 4-Hydroxyphenylammonium dihydrogen phosphate; C6 H8 N1 O1 1+,H2 O4 P1 1-; T.Glowiak, S.Debrus, M.May, A.J.Barnes, H.Ratajczak; J.Mol.Struct., 596, 77, 2001.

PUMQAP01 4-Dimethylaminopyridinium 4-nitrophenolate 4-nitrophenol; C7 H11 N2 1+,C6 H4 N1 O3 1-,C6 H5 N1 O3; N.Vembu, M.Nallu, E.C.Spencer, J.A.K.Howard; Acta Crystallogr.,Sect.E: Struct.Rep.Online, 59, o1192, 2003.

PUPYII 3-Phenyl-5a,6,7,8,9,9a-hexahydro-5H-1,5-benzodiazepin-1-ium picrate; C15 H19 N2 1+,C6 H2 N3 O7 1-; D.Lloyd, H.McNab, S.Parsons; J.Chem.Res., 70, 501, 1998.

PUPYOO 6-Phenyl-3,4-dihydro-2H-1,4-diazepin-1-ium picrate 1,2-dimethoxyethane solvate; C11 H13 N2 1+,C6 H2 N3 O7 1-,0.5(C4 H10 O2); D.Lloyd, H.McNab, S.Parsons; J.Chem.Res., 70, 501, 1998.

PUVMAU 5-Ammoniosalicylic acid chloride monohydrate; C7 H8 N1 O3 1+,Cl1 1-,H2 O1;

A.J.Dobson, R.E.Gerkin; Acta Crystallogr.,Sect.C: Cryst.Struct.Commun., 54, 1632, 1998.

PUVMIC bis(2,2'-Bipyridine) 1,3,5-trihydroxybenzene; 2(C10 H8 N2),C6 H6 O3;

E.S.Lavender, C.Glidewell, G.Ferguson; Acta Crystallogr.,Sect.C:

Cryst.Struct.Commun., 54, 1637, 1998.

PUVNID o-Phenylenediamine pyrocatechol hemihydrate; C6 H6 O2,C6 H8 N2,0.5(H2 O1);

S.Goswami, R.Mukherjee, G.D.Nigam, K.Chinnakali, Hoong-Kun Fun; Acta Crystallogr.,Sect.C: Cryst.Struct.Commun., 54, 1647, 1998.

PUYTAE Hydroquinone 3-methyl-4-nitropyridine 1-oxide; C6 H6 O2,C6 H6 N2 O3;

R.Moreno-Fuquen, A.M.Montano, R.Atencio; Acta Crystallogr.,Sect.E:

Struct.Rep.Online, 58, o623, 2002.

PYRPIC02 Pyridinium picrate; C5 H6 N1 1+,C6 H2 N3 O7 1-; M.Botoshansky, F.H.Herbstein, M.Kapon; Acta Crystallogr.,Sect.B: Struct.Sci., 50, 191, 1994. 
QAFZUT trans-1-(2-Pyridyl)-2-(4-pyridyl)ethylene 4-chlororesorcinol; C12 H10 N2,C6 H5 Cl1 O2; T.D.Hamilton, G.S.Papaefstathiou, L.R.MacGillivray; J.Am.Chem.Soc., 124, 11606, 2002.

QAGBAB 6-Amino-2,4-dinitrophenol; C6 H5 N3 O5; M.A.Menelaou, N.H.Fischer, F.R.Fronczek; Private Communication, , , 1999.

QENNEC Hydroquinone 2-hydroxy-6-isopropylcyclohepta-2,4,6-trien-1-one clathrate; C6 H6 O2,C10 H12 O2; K.Tanaka, R.Nagahiro, S.Ohba, M.Eishima; Tetrahedron Lett., 42, 925, 2001.

QEYCUS 5-Amino-2-pyrazolium picrate; C3 H6 N3 1+,C6 H2 N3 O7 1-; L.Infantes, C.FocesFoces, R.M.Claramunt, C.Lopez, J.Elguero; J.Heterocycl.Chem., 36, 595, 1999. QICWEE 2-t-Butyl-4,4,5,5-tetramethyl-1,2,3-triazolium picrate; C10 H22 N3 1+,C6 H2 N3 O7 1-; P.S.Engel, Li Pan, K.H.Whitmire, I.Guzman-Jimenez, M.R.Willcott, W.B.Smith; J.Org.Chem., 65, 1016, 2000.

QIJHUM 7-Methyl-1,5,7-triazabicyclo(4.4.0)dec-5-ene bis(pentachlorophenol); C8 H16 N3 1+,C6 Cl5 O1 1-,C6 H1 Cl5 O1; T.Glowiak, I.Majerz, L.Sobczyk, B.Brzezinski, G.Wojciechowski, E.Grech; J.Mol.Struct., 526, 177, 2000.

QIQLIL Benzocaine picrate; C9 H12 N1 O2 1+,C6 H2 N3 O7 1-; J.-O.Henck, J.Bernstein, A.Ellern, R.Boese; J.Am.Chem.Soc., 123, 1834, 2001.

QIQLIL01 benzocaine picrate; C9 H12 N1 O2 1+,C6 H2 N3 O7 1-; J.-O.Henck, J.Bernstein, A.Ellern, R.Boese; J.Am.Chem.Soc., 123, 1834, 2001.

QIQLOR bis(Benzocaine) picrate; C9 H12 N1 O2 1+,C9 H11 N1 O2,C6 H2 N3 O7 1-; J.O.Henck, J.Bernstein, A.Ellern, R.Boese; J.Am.Chem.Soc., 123, 1834, 2001. QIVTUK 2,4-Dihydroxybenzoic acid hemihydrate; C7 H6 O4,0.5(H2 O1); V.Horneffer, K.Dreisewerd, H.-C.Ludemann, F.Hillenkamp, M.Lage, K.Strupat; Int.J.Mass Spectrom.Ion.Process., 185, 859, 1999. 
QIWZOL 5-(2-Aminoethylthio)-2-methyl-1-phenylimidazole dipicrate; C12 H17 N3 S1

2+,2(C6 H2 N3 O7 1-); J.Suwinski, K.Swierczek, P.Wagner, M.Kubicki,

T.Borowiak; Pol.J.Chem., 75, 639, 2001.

QIXVUO 8-Hydroxyquinoline 2,4,5-trichlorophenol; C9 H7 N1 O1,C6 H3 Cl3 O1;

N.B.Singh, A.Srivastava, R.Frohlich; J.Chem.Soc.,Perkin Trans.2, , 838, 2001.

QOGMAA 1,2,4,5-Tetrahydroxybenzene monohydrate; C6 H6 O4,H2 O1; P.G.Jene,

C.G.Pernin, J.A.Ibers; Acta Crystallogr.,Sect.C: Cryst.Struct.Commun., 57, 730, 2001.

QOGMII 1,2,4,5-Tetrahydroxybenzene 2,5-dihydroxy-1,4-benzoquinone; C6 H6 O4,C6 H4

O4; P.G.Jene, C.G.Pernin, J.A.Ibers; Acta Crystallogr.,Sect.C:

Cryst.Struct.Commun., 57, 730, 2001.

QOGMII01 1,2,4,5-Tetrahydroxybenzene 2,5-dihydroxy-1,4-benzoquinone; C6 H6 O4,C6 H4

O4; P.G.Jene, C.G.Pernin, J.A.Ibers; Acta Crystallogr.,Sect.C:

Cryst.Struct.Commun., 57, 730, 2001.

QOYHAN 1-Azonia-4-azabicyclo(2.2.2)octane 3,5-dihydroxybenzoate monohydrate; C6 H13

N2 1+,C7 H5 O4 1-,H2 O1; C.J.Burchell, G.Ferguson, A.J.Lough, R.M.Gregson,

C.Glidewell; Acta Crystallogr.,Sect.B: Struct.Sci., 57, 329, 2001.

QOYHER Piperazinium bis(3,5-dihydroxybenzoate) tetrahydrate; C4 H12 N2 2+,2(C7 H5 O4

1-),4(H2 O1); C.J.Burchell, G.Ferguson, A.J.Lough, R.M.Gregson, C.Glidewell;

Acta Crystallogr.,Sect.B: Struct.Sci., 57, 329, 2001.

QUGWAQ $\quad \operatorname{rac}-\left(1 \mathrm{~S}^{*}, 1^{\prime} \mathrm{S}^{*}, 2^{\prime} \mathrm{S}^{*}\right)-1-(((2-\mathrm{Methoxy}-1-\mathrm{methyl}-2-$ phenylethoxy)methyl)dimethylsilyl)-

1-phenylethanol phenol solvate; C21 H30 O3 Si1,C6 H6 O1; S.Gassmann,

B.Guintchin, S.Bienz; Organometallics, 20, 1849, 2001.

QUOLUR Quinol-urea; C6 H6 O2,C1 H4 N2 O1; M.M.Mahmoud, S.C.Wallwork; Acta

Crystallogr.,Sect.B: Struct.Crystallogr.Cryst.Chem., 31, 338, 1975. 
RACBED 3-Hydroxypyridinium 2,5-dihydroxybenzoate; C5 H6 N1 O1 1+,C7 H5 O4 1-; T.Fukunaga, S.Kashino, H.Ishida; Acta Crystallogr.,Sect.E: Struct.Rep.Online, 59, o420, 2003.

RAKQAV 4-Methylpyridine pentachlorophenol; C6 Cl5 O1 1-,C6 H8 N1 1+; T.Steiner, I.Majerz, C.C.Wilson; Angew.Chem.,Int.Ed.Engl., 40, 2651, 2001.

RAKQAV01 4-Methylpyridine pentachlorophenol; C6 Cl5 O1 1-,C6 H8 N1 1+; T.Steiner, I.Majerz, C.C.Wilson; Angew.Chem.,Int.Ed.Engl., 40, 2651, 2001.

RAKQAV02 4-Methylpyridine pentachlorophenol; C6 Cl5 O1 1-,C6 H8 N1 1+; T.Steiner, I.Majerz, C.C.Wilson; Angew.Chem.,Int.Ed.Engl., 40, 2651, 2001.

RALPAV N-(3,4-Dichlorophenethyl)-N-methyl-N-(5-phenyl-1,2,4-oxadiazol-3ylmethyl)ammonium picrate; C18 H18 Cl2 N3 O1 1+,C6 H2 N3 O7 1-; C.Jager, C.Laggner, K.Mereiter, W.Holzer; Tetrahedron, 58, 10417, 2002.

RAWDIC tris(Hexamethylenetetramine) bis(1,3,5-trihydroxybenzene); 3(C6 H12 N4),2(C6 H6 O3); P.I.Coupar, C.Glidewell, G.Ferguson; Acta Crystallogr.,Sect.B: Struct.Sci., 53, $521,1997$.

REBFUZ bis(Triphenylphosphine oxide) tris(phenol); 2(C18 H15 O1 P1),3(C6 H6 O1); D.C.Apperley, P.A.Chaloner, L.A.Crowe, R.K.Harris, R.M.Harrison, P.B.Hitchcock, C.M.Lagier; Phys.Chem.Chem.Phys.(PCCP), 2, 3511, 2000.

REHNAT 1,2,3,4-Tetracyanobenzene p-hydroquinone; C10 H2 N4,C6 H6 O2; H.Bock, W.Seitz, M.Sievert, M.Kleine, J.W.Bats; Liebigs Ann., , 1929, 1996.

REHNEX N,N,N',N'-Tetramethylbenzidine bis(2,3,5,6-tetracyanohydroquinone) dihydrate; C16 H22 N2 2+,2(C10 H1 N4 O2 1-),2(H2 O1); H.Bock, W.Seitz, M.Sievert, M.Kleine, J.W.Bats; Liebigs Ann., , 1929, 1996.

REJMOI Methyl 2-(2-phenylbenzimidazolium-1-yl)propionate picrate; C17 H17 N2 O2 1+,C6 H2 N3 O7 1-; R.H.Jones, C.A.Ramsden, H.L.Rose; J.Heterocycl.Chem., 33, 1031, 1996. 
REPMUU 2,4,6-Trimethylpyridinium 2,6-dichloro-4-nitrophenolate; C8 H12 N1 1+,C6 H2 Cl2

N1 O3 1-; I.Majerz, W.Sawka-Dobrowolska, L.Sobczyk; Acta Phys.Pol.,A, 88, 349, 1995.

RESORA03 1,3-Benzenediol; C6 H6 O2; F.R.Fronczek; Private Communication, , , 2001.

RESORA13 Resorcinol; C6 H6 O2; G.E.Bacon, R.J.Jude;

Z.Kristallogr.,Kristallgeom.,Kristallphys.,Kristallchem., 138, 19, 1973.

RESVIU Dichloropicric acid; C6 H1 Cl2 N3 O7; M.K.Chantooni Junior, D.Britton;

J.Chem.Cryst., 27, 237, 1997.

ROHDIB 9a-Trimethylsilyloctahydroquinolizinium picrate; C12 H26 N1 Si1 1+,C6 H2 N3 O7

1-; J.M.White, Wai-Ying Tham; Acta Crystallogr.,Sect.C: Cryst.Struct.Commun., 53, 1471, 1997.

ROQLAK Morpholinium bis(2-chlorophenoxide); C4 H10 N1 O1 2+,2(C6 H4 Cl1 O1 1-);

M.A.Sridhar, J.S.Prasad, A.Pasha; Z.Kristallogr., 212, 389, 1997.

RUVVAF Hydroquinone bis(3,5-bis(2-pyridyl)-1,2,4-triazole); 2(C12 H9 N5),C6 H6 O2;

M.Nieuwenhuyzen, T.E.Keyes, J.F.Gallagher, J.G.Vos; Acta Crystallogr.,Sect.C:

Cryst.Struct.Commun., 53, 1873, 1997.

RUXDAP N-Methylmorpholine betaine 4-hydroxybenzoic acid; C7 H13 N1 O3,C7 H6 O3;

Z.Dega-Szafran, G.Dutkiewicz, Z.Kosturkiewicz, M.Przedwojska, M.Szafran;

J.Mol.Struct., 649, 257, 2003.

RUXDET 1,5-Diamino-1H-1,2,3,4-tetrazolium picrate; C6 H2 N3 O7 1-,C1 H5 N6 1+;

V.E.Matulis, A.S.Lyakhov, P.N.Gaponik, S.V.Voitekhovich, O.A.Ivashkevich;

J.Mol.Struct., 649, 309, 2003.

SAQJEZ 5-Ammoniosalicylium chloride; C7 H8 N1 O3 1+,Cl1 1-; Z.Banic-Tomisic,

B.Kojic-Prodic, I.Sirola; J.Mol.Struct., 416, 209, 1997. 
SAQJID 1-(Carboxymethyl)pyridinium 2,6-dichloro-4-nitrophenolate; C7 H8 N1 O2 1+,C6

H2 Cl2 N1 O3 1-; M.Szafran, Z.Dega-Szafran, G.Buczak, A.Katrusiak,

M.J.Potrzebowski, A.Komasa; J.Mol.Struct., 416, 145, 1997.

SARDAQ 1,4-Diaminobenzene diphenol; C6 H8 N2,2(C6 H6 O1); J.H.Loehlin, K.J.Franz,

L.Gist, R.H.Moore; Acta Crystallogr.,Sect.B: Struct.Sci., 54, 695, 1998.

SARDIY 1,4-Diaminobenzene bis(4-phenylphenol); C6 H8 N2,2(C12 H10 O1); J.H.Loehlin,

K.J.Franz, L.Gist, R.H.Moore; Acta Crystallogr.,Sect.B: Struct.Sci., 54, 695, 1998.

SARLEC 1,4-Diaminobenzene bis(4-chloro-1-hydroxybenzene); C6 H8 N2,2(C6 H5 Cl1 O1);

J.H.Loehlin, K.J.Franz, L.Gist, R.H.Moore; Acta Crystallogr.,Sect.B: Struct.Sci., 54, $695,1998$.

SAZGIJ 2,4-Dimethylpyridinium pentachlorophenolate; C7 H10 N1 1+,C6 Cl5 O1 1-;

I.Majerz, Z.Malarski, T.Lis; J.Crystallogr.Spectrosc.Res., 19, 349, 1989.

SENJOK 1,4-Dioxane hydroquinone; C4 H8 O2,C6 H6 O2; J.C.Barnes, J.D.Paton, C.S.Blyth; Acta Crystallogr.,Sect.C: Cryst.Struct.Commun., 46, 1183, 1990.

SEZREU Imidazolium picrate; C3 H5 N2 1+,C6 H2 N3 O7 1-; M.Soriano-Garcia, M.Schatz-

Levine, R.A.Toscano, R.V.Iribe; Acta Crystallogr.,Sect.C: Cryst.Struct.Commun., 46, 1556, 1990.

SMSALA01 4-Methyl-5-sulfosalicylic acid diaqua-oxonium monohydrate; C8 H7 O6 S1 1-,H3

O1 1+,3(H2 O1); T.Gustafsson; Acta Crystallogr.,Sect.C: Cryst.Struct.Commun., 41, 443, 1985.

SUFFOO 2,6-Dimethylbicyclo(3.3.1)nonane-exo-2,exo-6-diol p-chlorophenol clathrate; C11 H20 O2,C6 H5 Cl1 O1; A.T.Ung, R.Bishop, D.C.Craig, I.G.Dance, M.L.Scudder; Chem.Commun., , 322, 1993.

SUFFOO10 2,6-Dimethylbicyclo(3.3.1)nonane-exo-2,exo-6-diol p-chlorophenol clathrate; C11 H20 O2,C6 H5 Cl1 O1; A.T.Ung, R.Bishop, D.C.Craig, I.G.Dance, M.L.Scudder; Chem.Mater., 6, 1269, 1994. 
SUFFUU 2,6-Dimethylbicyclo(3.3.1)nonane-exo-2,exo-6-diol hydroquinone clathrate; C11 H20 O2,0.5(C6 H6 O2); A.T.Ung, R.Bishop, D.C.Craig, I.G.Dance, M.L.Scudder; Chem.Commun., , 322, 1993.

SUFFUU10 bis(2,6-Dimethylbicyclo(3.3.1)nonane-exo-2,exo-6-diol) hydroquinone clathrate; 2(C11 H20 O2),C6 H6 O2; A.T.Ung, R.Bishop, D.C.Craig, I.G.Dance, M.L.Scudder; Chem.Mater., 6, 1269, 1994.

SUNDUA 3-Acetyl-5-hydroxy-2-methylbenzaldehyde; C10 H10 O3; E.P.Kundig, A.Ripa, R.Liu, G.Bernardinelli; J.Org.Chem., 59, 4773, 1994.

SUVZEO 4-Nitropyridine N-oxide 3-hydroxybenzoic acid; C5 H4 N2 O3,C7 H6 O3;

R.Moreno-Fuquen, J.Valderrama-Naranjo, A.M.Montano; Acta Crystallogr.,Sect.C: Cryst.Struct.Commun., 55, 218, 1999.

SUWGOG bis(5-(2-Pyridyl)-1H-1,2,4-triazole) 1,4-dihydroxybenzene; 2(C7 H6 N4),C6 H6 O2;

M.Nieuwenhuyzen, R.Keirse, B.Shaw, J.G.Vos; Acta Crystallogr.,Sect.C:

Cryst.Struct.Commun., 55, 264, 1999.

TAHFIR 2,3-Dichloro-5,6-dicyano-1,4-dihydroxybenzene; C8 H2 Cl2 N2 O2; D.S.Reddy, Y.E.Ovchinnikov, O.V.Shishkin, Yu.T.Struchkov, G.R.Desiraju; J.Am.Chem.Soc., 118, 4085, 1996.

TAHFIR01 2,3-Dichloro-5,6-dicyano-1,4-dihydroxybenzene; C8 H2 Cl2 N2 O2; G.Hoshina, S.Ohba, M.Tsuchimoto; Acta Crystallogr.,Sect.C: Cryst.Struct.Commun., 55, 592, 1999.

TAHVII 1,2,4,5-tetrakis(2-(Pyrid-2-yl)ethynyl)benzene bis(resorcinol); C34 H18 N4,2(C6 H6 O2); E.Bosch, N.Schultheiss, N.Rath, M.Bond; Cryst.Growth Des., 3, 263, 2003.

TAPCES 1-Dimethylammonio-8-dimethylamino-naphthlene pentachlorophenolate bis(pentachlorophenol); C14 H19 N2 1+,C6 Cl5 O1 1-,2(C6 H1 Cl5 O1); J.A.Kanters, E.H.ter Horst, J.Kroon, E.Grech; Acta Crystallogr.,Sect.C: Cryst.Struct.Commun., 48, 328, 1992. 
TECHAK Dimethyl 4-hydroxy-6,6,7,7-tetramethyl-dehydroquinuclidinium-2,3-dicarboxylate picrate monohydrate; C15 H24 N1 O5 1+,C6 H2 N3 O7 1-,H2 O1;

R.G.Kostyanovsky, Y.I.El'natanov, I.I.Chervin, S.V.Konovalikhin, L.O.Atovmyan, A.Rauk; Mendeleev Commun., , 108, 1996.

TEFLUL DL-Arginine picrate; C6 H15 N4 O2 1+,C6 H2 N3 O7 1-; H.Nagata, Y.In, K.Tomoo, M.Doi, T.Ishida, A.Wakahara; Chem.Pharm.Bull., 43, 1836, 1995.

TELVIP p-Nitroaniline 3,5-dinitromethyl salicylate; C8 H6 N2 O7,C6 H6 N2 O2; Kin-Shan Huang, D.Britton, M.C.Etter; Acta Crystallogr.,Sect.C: Cryst.Struct.Commun., 52, 2868, 1996.

TEMWUD 2-Nitroresorcinol; C6 H5 N1 O4; C.R.Ojala, W.H.Ojala, D.Britton; J.Chem.Cryst., 26, 191, 1996.

TERSAK 4,6,8-Trimethylazulene picric acid; C13 H14,C6 H3 N3 O7; C.Nather, C.Arad, H.Bock; Acta Crystallogr.,Sect.C: Cryst.Struct.Commun., 53, 76, 1997.

TEXPOB Pyrene 2,3,5,6-tetracyanohydroquinone; C16 H10,C10 H2 N4 O2; H.Bock, W.Seitz, M.Sievert, M.Kleine, J.W.Bats; Angew.Chem.,Int.Ed.Engl., 35, 2244, 1996.

TEXPUH Perylene 2,3,5,6-tetracyanohydroquinone dihydrate; C20 H12,C10 H2 N4 O2,2(H2 O1); H.Bock, W.Seitz, M.Sievert, M.Kleine, J.W.Bats; Angew.Chem.,Int.Ed.Engl., $35,2244,1996$.

TICRUS o-Cyanophenol; C7 H5 N1 O1; C.Beswick, M.Kubicki, P.W.Codding; Acta Crystallogr.,Sect.C: Cryst.Struct.Commun., 52, 3171, 1996.

TICYOT 2,4,6-Trinitro-1,3,5-benzenetriol; C6 H3 N3 O9; J.J.Wolff, F.Gredel, H.Irngartinger, T.Dreier; Acta Crystallogr.,Sect.C: Cryst.Struct.Commun., 52, 3225, 1996. 
TIJKIG Hexahydroxybenzene bis(2,2'-bipyridine); C6 H6 O6,2(C10 H8 N2); J.A.Cowan, J.A.K.Howard, M.A.Leech, H.Puschmann, I.D.Williams; Acta Crystallogr.,Sect.C: Cryst.Struct.Commun., 57, 1194, 2001.

TOHGUS Imidazole-4-acetic acid picric acid; C5 H7 N2 O2 1+,C6 H2 N3 O7 1-; Y.In, H.Nagata, M.Doi, T.Ishida, A.Wakahara; Acta Crystallogr.,Sect.C: Cryst.Struct.Commun., 53, 367, 1997.

TOLCAY 2-Hydroxyisonaphthalic acid monohydrate; C8 H6 O5,H2 O1; E.Solari, A.Klose, C.Floriani, N.Re, A.Chiesi-Villa, C.Rizzoli; Polyhedron, 15, 4103, 1996.

TOLCAY01 2-Hydroxy-1,3-dicarboxybenzene monohydrate; C8 H6 O5,H2 O1; K.Mereiter, W.Mikenda, G.Reichl; Private Communication, , , 2001.

TPTPCM DL-Tryptophan picrate methanol solvate; C11 H13 N2 O2 1+,C6 H2 N3 O7 1-,C1 H4 O1; G.L.Gartland, G.R.Freeman, C.E.Bugg; Acta Crystallogr.,Sect.B: Struct.Crystallogr.Cryst.Chem., 30, 1841, 1974.

TRYPIC Tryptamine picrate; C10 H13 N2 1+,C6 H2 N3 O7 1-; G.L.Bartland, G.R.Freeman, C.E.Bugg; Acta Crystallogr.,Sect.B: Struct.Crystallogr.Cryst.Chem., 30, 1841, 1974.

UBUBIC 1,5-Dimethyl-3-(2-pyridyl)-6-oxoverdazyl hydroquionone; C9 H10 N5 O1,C6 H6 O2; R.G.Hicks, M.T.Lemaire, L.Ohrstrom, J.F.Richardson, L.K.Thompson, Zhiqiang Xu; J.Am.Chem.Soc., 123, 7154, 2001.

UDICEP 4-(N,N'-dimethylammonio)-5-(dimethylamino)-1-methyl-2-(2,2-dimethylpropyl)-2imidazolin-3-ium dipicrate; C13 H30 N4 2+,2(C6 H2 N3 O7 1-); S.Ohta, T.Osaki, S.Nishio, A.Furusawa, M.Yamashita, I.Kawasaki; Tetrahedron Lett., 41, 7503, 2000.

UFAZAC Piperazine-1,4-diium bis(2,4-dinitrophenolate) dihydrate; C4 H12 N2 2+,2(C6 H3 N2 O5 1-),2(H2 O1); A.Usman, S.Chantrapromma, Hoong-Kun Fun, Bo-Long Poh, C.Karalai; Acta Crystallogr.,Sect.C: Cryst.Struct.Commun., 58, o136, 2002. 
UHAHUG Triethyleneglycol dimethyl ether bis(dichloropicric acid) dihydrate; C8 H18 O4,2(C6 H1 Cl2 N3 O7),2(H2 O1); D.Britton, M.K.Chantooni Junior; J.Chem.Cryst., 31, 5, 2001.

UHAZOS bis(2,8-Dimethyltricyclo(7.1.1\$1,7!.1\$3,9!)dodecane-2,8-diol) hydroquinone; 2(C14 H24 O2),C6 H6 O2; Weimin Yue, R.Bishop, D.C.Craig, M.L.Scudder; CrystEngComm, 4, 591, 2002.

UHAZUY 2,5,8-Trimethyltricyclo(7.1.1\$1,7!.1\$3,9!)dodecane-2,8-diol hydroquinone; C15 H26 O2,C6 H6 O2; Weimin Yue, R.Bishop, D.C.Craig, M.L.Scudder; CrystEngComm, 4, 591, 2002.

UJOQUF 4,4'-Bipyridinium dipicrate monohydrate; C10 H10 N2 2+,2(C6 H2 N3 O7 1-),H2 O1; T.Akutagawa, T.Uchimaru, K.Sakai, T.Hasegawa, T.Nakamura; J.Phys.Chem.B, 107, 6248, 2003.

UJOREQ 4,4'-Bipyridine bis(2,5-dinitrophenol); C10 H8 N2,2(C6 H4 N2 O5); T.Akutagawa, T.Uchimaru, K.Sakai, T.Hasegawa, T.Nakamura; J.Phys.Chem.B, 107, 6248, 2003.

VAKTOR 4-Hydroxybenzoic acid isonicotinamide; C7 H6 O3,C6 H6 N2 O1; P.Vishweshwar, A.Nangia, V.M.Lynch; CrystEngComm, 5, 164, 2003.

VAKTUX Resorcinol bis(isonicotinamide); C6 H6 O2,2(C6 H6 N2 O1); P.Vishweshwar, A.Nangia, V.M.Lynch; CrystEngComm, 5, 164, 2003.

VAKVEJ Phloroglucinol bis(isonicotinamide) dihydrate; C6 H6 O3,2(C6 H6 N2 O1),2(H2 O1); P.Vishweshwar, A.Nangia, V.M.Lynch; CrystEngComm, 5, 164, 2003.

VAKVIN Hydroquinone bis(isonicotinamide); C6 H6 O2,2(C6 H6 N2 O1); P.Vishweshwar, A.Nangia, V.M.Lynch; CrystEngComm, 5, 164, 2003.

VATCOI bis(2,4-Dichloro-3-(pyrrolidinylmethylene)cyclohexa-1,4-diene-1,5dicarboxaldehyde) hydroquinone; 2(C13 H13 Cl2 N1 O2),C6 H6 O2; A.R.Katritzky, C.M.Marson, G.Palenik, A.E.Koziol, H.Luce, M.Karelson, Ban-Chi Chen, W.Brey; Tetrahedron, 44, 3209, 1988. 
VEVYAW 18-Crown-6 hydroquinone clathrate hexahydrate; C12 H24 O6,C6 H6 O2,6(H2 O1);

B.Belamri, C.Bavoux, A.Thozet; J.Inclusion Phenom.Macrocyclic Chem., 8, 383, 1990.

VICPOM 6-Furfurylpurine picrate; C10 H10 N5 O1 1+,C6 H2 N3 O7 1-; M.Soriano-Garcia, R.A.Toscano, G.Espinosa; J.Crystallogr.Spectrosc.Res., 15, 651, 1985.

VIMNOL10 (alphaS(R,R))-alpha-((Di-s-butylamino)methyl)-1-(2-chlorobenzyl)-1H-2-pyrrolemethanol p-hydroxybenzoate; C21 H32 Cl1 N2 O1 1+,C7 H5 O3 1-; J.V.Silverton, H.A.Lloyd; Acta Crystallogr.,Sect.B: Struct.Crystallogr.Cryst.Chem., 31, 1576, 1975.

VIVYUU cis-trans-Diacetamide 4-nitrophenol clathrate; C6 H5 N1 O3,C4 H7 N1 O2;

M.C.Etter, S.M.Reutzel; J.Am.Chem.Soc., 113, 2586, 1991.

VIVZAB N-Butyrylbenzamide 4-nitrophenol clathrate; C11 H13 N1 O2,C6 H5 N1 O3;

M.C.Etter, S.M.Reutzel; J.Am.Chem.Soc., 113, 2586, 1991.

VIVZEF bis(Diacetamide) hydroquinone clathrate; C6 H6 O2,2(C4 H7 N1 O2); M.C.Etter, S.M.Reutzel; J.Am.Chem.Soc., 113, 2586, 1991.

VIVZIJ bis(N-Butyrylbenzamide) hydroquinone clathrate; 2(C11 H13 N1 O2),C6 H6 O2; M.C.Etter, S.M.Reutzel; J.Am.Chem.Soc., 113, 2586, 1991.

VIVZOP Diacetamide 4-hydroxybenzoic acid clathrate; C7 H6 O3,C4 H7 N1 O2; M.C.Etter, S.M.Reutzel; J.Am.Chem.Soc., 113, 2586, 1991.

VIVZUV Diacetamide 4-hydroxybenzamide clathrate; C7 H7 N1 O2,C4 H7 N1 O2; M.C.Etter, S.M.Reutzel; J.Am.Chem.Soc., 113, 2586, 1991.

VOQLIW bis(Cephradine) 4-hydroxybenzoic acid tetrahydrate; 2(C16 H19 N3 O4 S1),C7 H6 O3,4(H2 O1); G.J.Kemperman, R.de Gelder, F.J.Dommerholt, P.C.RaemakersFranken, A.J.H.Klunder, B.Zwanenburg; Eur.J.Org.Chem., , 3641, 2001. 
VUXJUT 1,4,7,10,13,16-Hexaoxacyclo-octadecane 2-isopropyl-3-methylphenol dihydrate;

C12 H24 O6,2(C10 H14 O1),2(H2 O1); B.Belamri, C.Bavoux; Acta

Crystallogr.,Sect.C: Cryst.Struct.Commun., 44, 2173, 1988.

VUXXUH Diaza-18-crown-6 2-hydroxy-5-methyl-1,3-benzenedicarbaldehyde; C12 H28 N2

O4 2+,2(C9 H7 O3 1-); W.H.Watson, A.Nagl, F.Vogtle, W.M.Muller; Acta

Crystallogr.,Sect.C: Cryst.Struct.Commun., 44, 1499, 1988.

WADGEO Methyl 2,5-dihydroxybenzoate; C8 H8 O4; C.L.Brown, J.F.Heagney, D.J.Young,

P.C.Healy; Acta Crystallogr.,Sect.E: Struct.Rep.Online, 59, o630, 2003.

WIRWID 2-Picoline N-oxide 4-nitrophenol; C6 H7 N1 O1,C6 H5 N1 O3; R.Moreno-Fuquen,

M.T.do P.Gambardella, R.H.de Almeida Santos; Acta Crystallogr.,Sect.C:

Cryst.Struct.Commun., 52, 1745, 1996.

WOCRIP 1,4-Diazabicyclo(2.2.2)octane 5-hydroxyisophthalic acid; C6 H13 N2 1+,C8 H5 O5

1-; C.J.Burchell, G.Ferguson, A.J.Lough, C.Glidewell; Acta Crystallogr.,Sect.C:

Cryst.Struct.Commun., 56, 1126, 2000.

WOKGEI 2-Hydroxy-7-oxa-4-azoniaspiro(3.5)nonane picrate; C7 H14 N1 O2 1+,C6 H2 N3

O7 1-; V.N.Knyazev, O.Ya.Borbulevych, O.V.Shishkin;

Zh.Org.Khim.(Russ.)(Russ.J.Org.Chem.), 36, 733, 2000.

WOLZUS Trimethylamine N-oxide pentachlorophenol; C3 H10 N1 O1 1+,C6 Cl5 O1 1-;

E.Tykarska, Z.Dega-Szafran, M.Szafran; J.Mol.Struct., 477, 49, 1999.

WOMBAB Trimethylamine N-oxide pentachlorophenol monohydrate; C3 H10 N1 O1 1+,C6

Cl5 O1 1-,H2 O1; E.Tykarska, Z.Dega-Szafran, M.Szafran; J.Mol.Struct., 477, 49, 1999.

XAGFAM Guanidine 2-hydroxybenzoic acid; C1 H6 N3 1+,C7 H5 O3 1-; M.Fleck,

E.Tillmanns, S.Haussuhl; Z.Kristallogr.-New Cryst.Struct., 215, 105, 2000.

XAXSIY 2,4,6-Trimethylphenol; C9 H12 O1; B.Ziemer, O.Surygina; Acta

Crystallogr.,Sect.C: Cryst.Struct.Commun., 56, e528, 2000. 
XAYCIJ 3-Hydroxybenzaldehyde; C7 H6 O2; J.A.Paixao, A.M.Beja, M.R.Silva, L.A.da Veiga, A.C.Serra; Acta Crystallogr.,Sect.C: Cryst.Struct.Commun., 56, 1348, 2000.

XEHJUP meso-5,5,7,12,12,14-Hexamethyl-1,4,8,11-tetra-azacyclotetradecane 5hydroxyisophthalic acid monohydrate; C16 H38 N4 2+,C8 H4 O5 2-,H2 O1; C.J.Burchell, G.Ferguson, A.J.Lough, C.Glidewell; Acta Crystallogr.,Sect.B: Struct.Sci., 56, 1054, 2000.

XEHKAW rac-5,5,7,12,12,14-Hexamethyl-1,4,8,11-tetra-azacyclotetradecane 5hydroxyisophthalic acid monohydrate; C16 H38 N4 2+,C8 H4 O5 2-,H2 O1; C.J.Burchell, G.Ferguson, A.J.Lough, C.Glidewell; Acta Crystallogr.,Sect.B: Struct.Sci., 56, 1054, 2000.

XETWIC 3,6-Dioxaoctane-1,8-diammonium dipicrate; C6 H18 N2 O2 2+,2(C6 H2 N3 O7 1-); A.Szumna, J.Jurczak, Z.Urbanczyk-Lipkowska; J.Mol.Struct., 526, 165, 2000.

XETWOI 3-Azapentane-1,5-diammonium dipicrate; C4 H15 N3 2+,2(C6 H2 N3 O7 1-); A.Szumna, J.Jurczak, Z.Urbanczyk-Lipkowska; J.Mol.Struct., 526, 165, 2000.

XEVRUL 2,4-Dihydroxybenzaldehyde; C7 H6 O3; Zhiyong Hu, M.J.Hardie, P.Burckel, A.A.Pinkerton, P.W.Erhardt; J.Chem.Cryst., 29, 185, 1999.

XIBQUU bis(1,3,5-Benzenetricarboxylic acid) 1,3,5-trihydroxybenzene; 2(C9 H6 O6),C6 H6 O3; Rong Liu, Kum-Fun Mok, S.Valiyaveettil; New J.Chem.(Nouv.J.Chim.), 25, 890, 2001.

XINHOR 1,10-Diazonia-18-crown-6 dipicrate; C12 H28 N2 O4 2+,2(C6 H2 N3 O7 1-); A.N.Chekhlov; Zh.Strukt.Khim.(Russ.)(J.Struct.Chem.), 42, 1016, 2001.

XOKBOO bis(4-Methylphenol) piperazine; 2(C7 H8 O1),C4 H10 N2; Zhimin Jin, Duanjun Xu, Yuanjiang Pan, Yuanzhi Xu, M.Yen-Nan Chiang; J.Mol.Struct., 559, 1, 2001. XUGPAQ 2,2'-(4,4'-Bipyridinium-1,1'-diyl)-bis(ethanesulfonate) bis(hydroquinone); C14 H16 N2 O6 S2,2(C6 H6 O2); P.D.Robinson, K.R.Smith, L.A.Vermeulen; Acta Crystallogr.,Sect.C: Cryst.Struct.Commun., 58, o632, 2002. 
XUTCAQ Piperazinedi-ium bis(4-nitrophenolate) dihydrate; C4 H12 N2 2+,2(C6 H4 N1 O3 1),2(H2 O1); Ya Xiong, Taicheng An, Chun He, Yi Liu, Jiang Chen, Changhong Zha, H.T.Karlsson, Xiaoming Chen; J.Chem.Cryst., 32, 219, 2002.

XUTCEU Piperazinedi-ium bis(2-nitrophenolate) dihydrate; C4 H12 N2 2+,2(C6 H4 N1 O3 1),2(H2 O1); Ya Xiong, Taicheng An, Chun He, Yi Liu, Jiang Chen, Changhong Zha, H.T.Karlsson, Xiaoming Chen; J.Chem.Cryst., 32, 219, 2002.

YAVDOO Ammonium catecholate catechol hemihydrate; C6 H5 O2 1-,C6 H6 O2,H4 N1 1+,0.5(H2 O1); O.Kumberger, J.Riede, H.Schmidbaur; Z.Naturforsch.,B:

Chem.Sci., 48, 958, 1993.

YAXCEF01 Pentaethyleneglycol dimethyl ether bis(dichloropicric acid) dihydrate; C12 H26 O6,2(C6 H1 Cl2 N3 O7),2(H2 O1); M.K.Chantooni Junior, D.Britton, I.M.Kolthoff; J.Crystallogr.Spectrosc.Res., 23, 497, 1993.

YEJZAO Guanidinium 3,5-dinitrosalicylate; C1 H6 N3 1+,C7 H3 N2 O7 1-; G.Smith, R.C.Bott, U.D.Wermuth; Acta Crystallogr.,Sect.E: Struct.Rep.Online, 57, o640, 2001.

YIDKEB 4-Methylpyridinium 2,6-dichloro-4-nitrophenolate; C6 H8 N1 1+,C6 H2 Cl2 N1 O3 1-; I.Majerz, W.Sawka-Dobrowolska, L.Sobczyk; Pol.J.Chem., 67, 1657, 1993. YIJVOC Benzyldimethylammonium picrate; C9 H14 N1 1+,C6 H2 N3 O7 1-; V.A.Pankratov, T.M.Frenkel, A.E.Shvorak, S.V.Lindeman, Yu.T.Struchkov; Izv.Akad.Nauk SSSR,Ser.Khim.(Russ.)(Russ.Chem.Bull.), , 92, 1993.

YOLQIZ 3,5,7-Triaza-1-azoniatricyclo(3.3.1.1\$3,7!)decane 2,4,6-trinitrophenolate; C6 H13 N4 1+,C6 H2 N3 O7 1-; A.Usman, S.Chantrapromma, Hoong-Kun Fun, Bo-Long Poh, C.Karalai; Acta Crystallogr.,Sect.C: Cryst.Struct.Commun., 58, o46, 2002. YUJFIS bis(4-Hydroxyphenylammonium) dihydrogen diphosphate monohydrate; 2(C6 H8 N1 O1 1+),H2 O7 P2 2-,H2 O1; E.H.Soumhi, T.Jouini; Acta Crystallogr.,Sect.C: Cryst.Struct.Commun., 51, 1213, 1995. 
YUYLAF 4-Chloro-3,5-dimethylphenol; C8 H9 Cl1 O1; P.J.Cox; Acta Crystallogr.,Sect.C: Cryst.Struct.Commun., 51, 1361, 1995.

ZAJGUM 3,5-Dinitrosalicylic acid monohydrate; C7 H4 N2 O7,H2 O1; G.Smith, D.E.Lynch, K.A.Byriel, C.H.L.Kennard; Aust.J.Chem., 48, 1133, 1995.

ZAJHEX 3,5-Dinitrosalicylic acid 3-aminobenzoic acid; C7 H3 N2 O7 1-,C7 H8 N1 O2 1+; G.Smith, D.E.Lynch, K.A.Byriel, C.H.L.Kennard; Aust.J.Chem., 48, 1133, 1995.

ZAJHIB 3,5-Dinitrosalicylic acid bis(4-aminobenzoic acid); C7 H3 N2 O7 1-,C7 H7 N1 O2,C7 H8 N1 O2 1+; G.Smith, D.E.Lynch, K.A.Byriel, C.H.L.Kennard; Aust.J.Chem., 48, 1133, 1995.

ZAPFAX Hydroquinone hydrazine clathrate; C6 H6 O2,H4 N2; F.Toda, S.Hyoda, K.Okada, K.Hirotsu; Chem.Commun., , 1531, 1995.

ZEDPON01 2-Iodoanilinium picrate; C6 H2 N3 O7 1-,C6 H7 I1 N1 1+; M.Tanaka, H.Matsui, J.I.Mizoguchi, S.Kashino; Bull.Chem.Soc.Jpn., 67, 1572, 1994.

ZEZHIV L-Proline 2,5-dihydroxybenzoic acid; C5 H9 N1 O2,C7 H6 O4; C.B.Aakeroy, G.S.Bahra, C.R.Brown, P.B.Hitchcock, Y.Patell, K.R.Seddon; Acta Chem.Scand., 49, 762, 1995.

ZILLUB Indole-3-acetamide picric acid; C10 H10 N2 O1,C6 H3 N3 O7; H.Nagata, Y.In, M.Doi, T.Ishida, T.Ishida, A.Wakahara; Acta Crystallogr.,Sect.B: Struct.Sci., 51, 1051, 1995.

ZIVROL 3-Amino-2H,4H-1,2,4-triazolium 5-nitrosalicylate; C2 H5 N4 1+,C7 H4 N1 O5 1-; G.Smith, D.E.Lynch, K.A.Byriel, C.H.L.Kennard; Acta Crystallogr.,Sect.C: Cryst.Struct.Commun., 52, 231, 1996.

ZOCVES 3-Amino-1-phenyl-4,5-dihydropyrazol-2-ium picrate; C9 H12 N3 1+,C6 H2 N3 O7 1-; R.M.Claramunt, P.Cozzini, P.Domiano, J.Elguero, I.Forfar, A.Fruchier; J.Chem.Soc.,Perkin Trans.2, , 1875, 1995. 
ZOKSUN 4-N,N-Dimethylaminopyridinium pentachlorophenolate; C7 H11 N2 1+,C6 Cl5 O1

1-; I.Majerz, Z.Malarski, W.Sawka-Dobrowolska; J.Chem.Cryst., 25, 189, 1995.

ZOKYON Triethyleneglycol dimethyl ether bis(dichloropicric acid); 2(C6 H1 Cl2 N3 O7),C8

H18 O4; M.K.Chantooni Junior, D.Britton; J.Chem.Cryst., 25, 351, 1995.

ZUHKUI 3-Amino-4-hydroxybenzenesulfonic acid hemihydrate; C6 H7 N1 O4 S1,0.5(H2

O1); B.J.Gunderman, P.J.Squattrito; Acta Crystallogr.,Sect.C:

Cryst.Struct.Commun., 52, 940, 1996.

ZUWMAF 2-Ethylpyridinium 2,6-dichloro-4-nitrophenolate; C7 H10 N1 1+,C6 H2 Cl2 N1 O3

1-; I.Majerz, W.Sawka-Dobrowolska; J.Chem.Cryst., 26, 147, 1996.

ZZZLYS01 4,6-Dinitroresorcinol; C6 H4 N2 O6; T.Kolev, M.Berkei, C.Hirsch, H.Preut, P.Bleckmann, V.Radomirska; Z.Kristallogr.-New Cryst.Struct., 215, 483, 2000.

ZZZVLG11 Hydroquinone hydrogen sulfide clathrate; 3(C6 H6 O2),0.87(H2 S1); W.C.Ho, T.C.W.Mak; Z.Kristallogr.,Kristallgeom.,Kristallphys.,Kristallchem., 161, 87, 1982. 